\title{
Assessing the Feasibility of Replacing Standard-Dose Bacillus Calmette-Guérin Immunotherapy with Other Intravesical Instillation Therapies in Bladder Cancer Patients: A Network Meta-Analysis
}

\author{
Cheng Wu ${ }^{a}$ Xunrong Zhou ${ }^{a}$ Chunqing Miao ${ }^{a}$ Jianzhong Zhang ${ }^{b}$ \\ Qingsheng Tang ${ }^{\mathrm{a}}$ Xiumei Chang ${ }^{\mathrm{a}}$ Haodong $\mathrm{Ni}^{\mathrm{a}}$ \\ ${ }^{a}$ Department of Urology, People's Hospital of Dongtai City, Dongtai, bDepartment of Urology, The first \\ affiliated hospital of Nanjing Medical University, Nanjing, China
}

\section{Key Words}

Intravesical instillation therapies - Bacillus Calmette-Guérin • Mitomycin C • Epirubicin • Interferon-Alpha $\cdot$ Gemcitabine $\cdot$ Tumor recurrence $\cdot$ Tumor progression • Pairwise/network meta-analysis $\bullet$ Heterogeneity $\bullet$ Consistency

\begin{abstract}
Background: Bacillus Calmette-Guérin (BCG) immunotherapy plays a key role in patients with bladder cancer. The shortage of intravesical BCG has motivated researchers to seek alternatives with equivalent efficacy. If other alternative intravesical agents have equivalent efficacy compared to BCG, then it may be feasible to replace standard BCG with alternative options. Methods: We searched all relevant evidence in multiple sources and key data was extracted from included studies. Conventional and network meta-analysis were conducted so that pooled odds ratios (ORs) for the event of tumor recurrence and progression can be computed. The relative efficacy of different intravesical instillation procedures was computed by pooled odds ratios and their $95 \%$ confidence or creditable intervals. Besides, several key model assumptions were evaluated in our analysis. Results: Three intravesical instillation procedures have the potential for preventing tumor recurrence: standard-dose BCG (BCG_SD), Epirubicin (EPI) and Mitomycin C (MMC) (ORs < 1). Patients with BCG_SD also exhibited a decreased risk of tumor recurrence and progression compared to those with EPI. No significant difference in the risk of tumor recurrence or progression was detected between patients treated with BCG SD and those with low-dose BCG (BCG_LD). Results of SUCRA indicated that BCG_EPI, BCG_ $M M C$ and BCG_SD had higher rankings with respect to tumor recurrence and progression. Conclusions: BCG_SD, EPI and MMC exhibited established efficacy for preventing tumor recurrence in postoperative BC patients. The efficacy of BCG may not be significantly reduced if standard dose was reduced to a lower level. However, there is no consensus suggesting that intravesical BCG with standard dose can be replaced by alternating or sequentially combined intravesical instillation therapies.

(C) 2017 The Author(s) C. Wu and X. Zhou contributed equally to the study.




\section{Cellular Physiology Cell Physiol Biochem 2017;41:1298-1312 \begin{tabular}{ll|l} 
and Biochemistry & $\begin{array}{l}\text { DOI: 10.1159/000464432 } \\
\text { Publisned onlnne: IVIarch 08, } 2017\end{array}$ & $\begin{array}{l}\text { @ 2017 The Author(s). Published by S. Karger AG, Basel } \\
\text { www.karger.com/cpb }\end{array}$ \\
\hline
\end{tabular} \\ Wu et al.: Assessment of Therapies in Bladder Cancer}

\section{Introduction}

Bladder cancer (BC) is generally classified as starting from the urothelium. Although the exact cause of $\mathrm{BC}$ has not yet been identified, it is still associated with exposure to certain aromatic chemicals such as aniline dyes and benzidine compounds [1]. The survival rate of bladder cancer patients varies significantly with tumor stages. According to the American Cancer Society (ACS), the five-year survival rate decreased dramatically from $88 \%$ (stage I) to $15 \%$ (stage IV).

If tumors are diagnosed through pathological examinations, transurethral resection of bladder tumor (TURBT) will be performed. Treatment selection is determined by several factors: pathological classification, tumor staging and metastatic status. Immunotherapy using intravesical delivery of Bacillus Calmette-Guérin (BCG) plays a pivotal role in bladder cancer since it is effective in $\mathrm{BC}$ treatment and tumor recurrence prevention after surgical resection in a certain degree [1].

There is a potential shortage of intravesical BCG due to its increasing demand together with insufficient supplies [2]. Two potential solutions can be provided as treatment supplementary, particularly for patients with non-muscle-invasive bladder cancer. One typical approach is to use alternative intravesical agents instead, such as Mitomycin C (MMC), Epirubicin (EPI), Interferon-Alpha (INF) and Gemcitabine (GEM), which can be quite controversial in the meanwhile because it is believed immunotherapy of BCG can overcome short remissions resulted from intravesical chemotherapeutic agents [3, 4]. However, it can still be improved. For instance, intravesical BCG can be combined with other intravesical agents to enhance its efficacy in the long term. The second approach comes from the hypothesis that a lower dose of intravesical BCG has no significant influence on the overall efficacy [5]. This inevitably increases the complexity of evidence.

If the shortage of intravesical BCG production continues and the number of bladder cancer patients keeps increasing, evaluating the two solutions mentioned above will become an urgent task. Besides that, preventing the abuse of intravesical BCG is highly advocated, particularly for the non-muscle invasive bladder cancer (NMIBC) patients [6]. This network meta-analysis (NMA) was designed to compare the long-term efficacy of different intravesical instillation therapies. Several intravesical instillation therapies with different features (immunotherapy of intravesical BCG: standard dosage or reduced dosage; other intravesical instillation agents: MMC, EPI, INF, GEM; single, sequentially combined or alternating) are considered throughout the research protocol, systematic review and NMA.

\section{Material and Methods}

\section{Research protocol: systematic review and planning}

We deigned a study protocol to ensure the accuracy and feasibility of our study. The corresponding study protocol concerned about specific study populations, interventions, comparators and outcomes (PICOS). Besides that, a few items were discussed by the researchers in the protocol: search strategy, predefined study inclusion criteria, data extraction process, quality assessment, risk of bias and statistical approaches. Also, the implementation of our network meta-analysis was guided by the research protocol. A few meta-analyses were reviewed for reference. We also planned for some potential changes during the course of our research.

\section{Evidence searching}

An initial search was conducted in the current literature to ascertain that our research topics have not been studied. Multiple sources were identified and selected for preventing selection and reporting bias: Embase, PubMed, Cochrane Library and China National Knowledge Infrastructure (CNKI). Preliminary searching terms and strings were developed using the following key words: Urinary Bladder Neoplasms; Bladder Instillations; BCG Vaccine; Mutamycin; Epilem; Interferons; gemcitabine.. The entire searching strategy was conducted iteratively and the corresponding searching results were peer viewed for the purpose of quality control. Apart from the initial search, evidence searching was updated at the final stage. 


\section{Cellular Physiology Cell Physiol Biochem 2017;41:1298-1312 \begin{tabular}{l|l|l}
\hline DOI: 10.1159/000464432 & (c) 2017 The Author(s). Published by S. Karger AG, Basel
\end{tabular} and Biochemistry Publisned online: VVarcn 08, 2017 www.karger.com/cpb}

Wu et al.: Assessment of Therapies in Bladder Cancer

Eligibility criteria and study selection

Eligibility criteria were clearly defined before study selection. The corresponding eligibility criteria consider four key elements in order to ensure the validity of the upcoming network meta-analysis: types of studies, types of participants, types of interventions and types of outcomes. 1) Types of studies: randomized clinical trials (RCTs) comparing the efficacy or safety of intravesical agents for bladder cancer patients; trials were conducted between the year of 1986 and 2016 with a minimum follow-up duration of six months. 2) types of participants: postoperative (TUR) adult patients with proven evidence of non-muscle-invasive bladder cancer (with or without carcinoma in situ), superficial bladder cancer, recurrent bladder cancer, superficial bladder carcinoma patients with increased risk of tumor recurrence or progression, patients with previous BCG treatment or chemotherapy or radiation therapy, patients with severe infection or active tuberculosis, patients with other neoplastic diseases, lower urinary tract diseases or major concomitant diseases were excluded. 3) Types of interventions: instillation therapy with BCG (standard dose: $80 \mathrm{mg}$; low dose: $40 \mathrm{mg}$ ), MMC (10 mg, $30 \mathrm{mg}, 40 \mathrm{mg}$ ), EPI (20 mg, $40 \mathrm{mg}, 50 \mathrm{mg}, 80 \mathrm{mg}$ ), IFN (50 million units) and GEM (2,000 mg); trials with single, combined or alternating instillation therapy were all eligible. 4) Types of outcomes: trials that assessed intravesical instillation therapy by using tumor recurrence or tumor progression. The above eligibility criteria were also reviewed by senior researchers. Discussion was held in a group meeting if there was any disagreement.

\section{Data collection process}

We developed a preliminary data extraction sheet and identified a list of essential variables for our network meta-analysis. Then, a pilot data extraction was conducted by drawing a random sample from the eligible list. The initial data extraction was refined to reflect the nature of our network meta-analysis and the quality of included studies. The refined data extraction sheet covers three types of information for each eligible study: basic study information (author, publication year, average age of patients, the proportion of male patients, the following-up duration), comparators and outcomes (instillation therapy, sample size in each group, the number or proportion of patients who experienced tumor recurrence or progression by the end of the following-up period) and quality of eligible studies (appropriate randomization techniques, blinding procedures and disclosure of withdrawals). All study variables were clearly coded before data extraction. Eligible studies with missing variables were not removed in order to prevent selection bias. Missing study variables were not imputed (NR: Not Reported) if they cannot be estimated or computed from the original study. Data collection results were verified by two independent reviewers. All inconsistency was resolved by consensus before the commencement of statistical analysis.

\section{Quality of included studies}

The eligible study list was reviewed by our review team and the content for each study was carefully examined. Each included study was discussed based on the following topics: the adequacy of randomization, blinding procedures, the issue of loss to follow-up and the degree of heterogeneity among included studies. Then, the Jadad scale was used by two independent reviewers to quantify the quality of studies. Some potential sensitivity or subgroup analysis was also planned in this stage.

\section{Statistical analysis}

The efficacy of intravesical instillation therapies was compared by using the endpoint of tumor recurrence and progression. First, our reviewers performed a pairwise meta-analysis based on the fixedeffect model. The statistic of odds ratio (OR) for the event of tumor recurrence or progression was produced in each included study. Then, a weighted odds ratio together with its $95 \%$ confidence interval (95\% CI) was computed by the fixed-effect model for each direct comparison. Study heterogeneity within each direct comparison was measured by the statistics of $I^{2}$. The assumption of homogeneous effect size was tested by the Cochran's $Q$ statistics. Study heterogeneity is significant if $I^{2}>50 \%$ or $P$-value for the hypothesis testing of homogeneous effect < 0.1. After that, an NMA (random-effects model, Bayesian Framework) along with the Markov Chain Monte Carlo (MCMC) simulation was carried out to synthesize all relevant evidence. Summary OR and its 95\% credible interval (95\% CrI) for the event of tumor recurrence or progression were produced for comparison. Evidence inconsistency for each pair of comparison was assessed by the nodesplitting method [7]. Evidence was significantly inconsistent if the P-value of the node-splitting method $<0.05$. Intravesical instillation therapies were compared according to their surface under the cumulative 


\section{Cellular Physiology Cell Physiol Biochem 2017;41:1298-1312 \begin{tabular}{ll|l} 
and Biochemistry $10.1159 / 000464432$ & $\begin{array}{l}\text { P } 2017 \text { The Author(s). Published by S. Karger AG, Basel } \\
\text { www.karger.com/cpb }\end{array}$ \\
\hline
\end{tabular} \\ Wu et al.: Assessment of Therapies in Bladder Cancer}

ranking curve (SUCRA) values. Finally, funnel plots were created for detecting asymmetry patterns which are typical signs for publication bias. All of the above data manipulation and statistical analysis was implemented by using the statistical computing software R (The R Project for Statistical Computing, R version 3.2.5). The GEMTC package was also loaded into R software for Bayesian network meta-analysis and MCMC simulation.

\section{Results}

Literature search results

We initially identified 2,226 literature records from a variety of sources. All identified records were retrieved and imported into the bibliography management software (Endnote 7). Then, 248 duplicated or overlapped records were removed from the bibliography software. By reviewing the title and abstract of the remaining 1978 articles, we further excluded 1,909 studies that were not related to our research topics or did not fulfill the predefined eligibility criteria. As a result, only 69 studies were remained for full-text assessment. We identified and excluded 12 studies without full-text content or sufficient data during the course of full-text review. The above steps were performed by two independent literature searchers who did not conduct the previous literature search. Any discrepancy of the literature search results was discussed with caution (Fig. 1).

Characteristics of included studies and structures of network

In total, we included 57 RCTs [8-64] conducted between 1988 and 2016 (Table 1). All included studies were followed up for a minimum and maximum duration of 0.9 and 9.2 years, respectively. The 57 eligible studies included a total of 12,943 patients who were diagnosed with superficial bladder cancer, carcinoma in site or non-muscle invasive bladder cancer. There appeared to be significant variation in the demographic features of patients in each included study. For instance, the average age of patients ranged from 55 to 71.9 and some studies did not report the average age of patients. A vivid network demonstrating the pattern of evidence and comparisons is displayed in Fig. 2. The structure of evidence was formed by a total of 9 intravesical instillation therapies: BCG_SD (standard dose), BCG_MMC, BCG_LD (low dose), BCG_EPI, MMC, IFN, GEM and EPI. As shown in the network plot, the sample size for each intravesical instillation therapy varied remarkably.

\section{Pairwise meta-analysis}

The following groups of patients were exhibited a lower risk of tumor recurrence compared to those with placebo: BCG_SD (OR $=0.48,95 \% \mathrm{CI}=0.34-0.70)$, EPI $(\mathrm{OR}=$ $0.76,95 \% \mathrm{CI}=0.62-$ $0.94)$ and $\mathrm{MMC}(\mathrm{OR}=$ $0.65,95 \% \mathrm{CI}=0.48$ 0.88; Table 2). Direct evidence also revealed that postoperative $\mathrm{BC}$ patients who received BCG_MMC exhibited a reduced risk of tumor recurrence than those with BCG_SD $(\mathrm{OR}=0.74$, 95\% CI $=0.54-0.99$ ). By contrast, patients with EPI exhibited an elevated risk of tumor recurrence in relation to those with BCG_SD $(\mathrm{OR}=1.53,95 \% \mathrm{CI}=$

Fig. 1. Flow chart of the study selection.

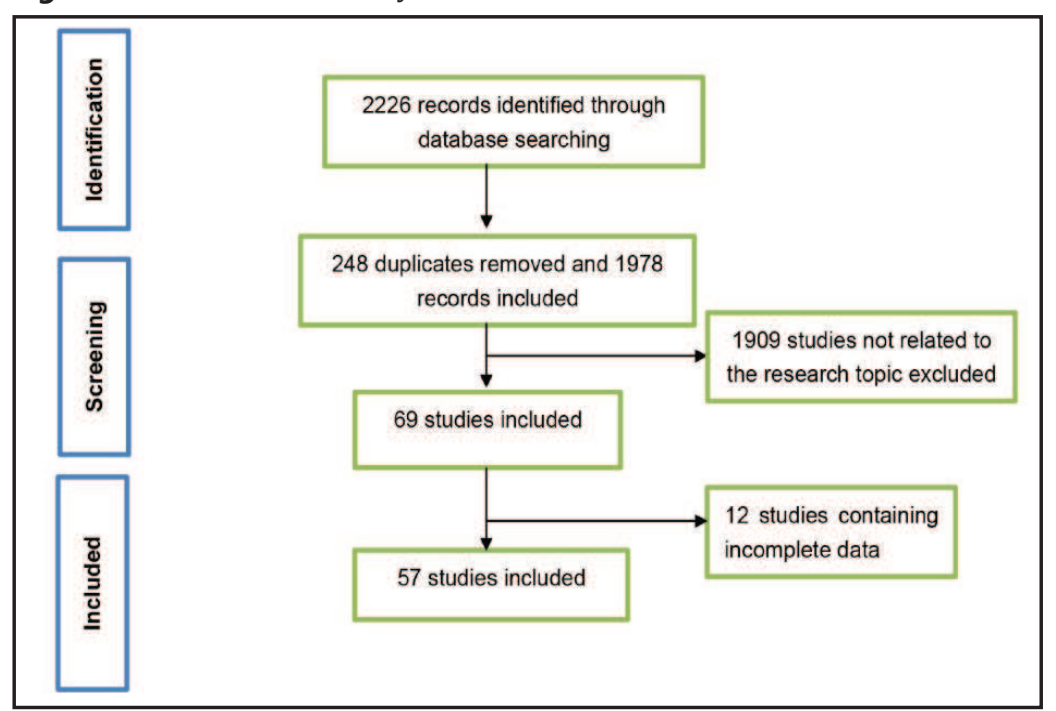



Cellular Physiology Cell Physiol Biochem 2017;41:1298-1312

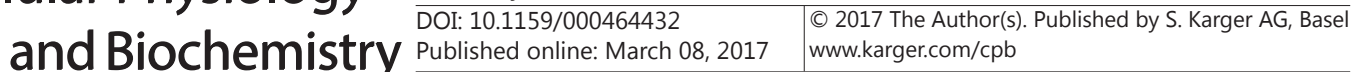

Wu et al.: Assessment of Therapies in Bladder Cancer

Table 1. The main characteristics of the included clinical trials. * NR: Not reported; BCG_SD: Bacille Calmette Guerin standard dose; BCG_LD: Bacille Calmette Guerin low dose; MMC: Mitomycin C; GEM: Gemcitabine; EPI: Epirubicin; IFN: Interferon

\begin{tabular}{|c|c|c|c|c|c|c|c|c|c|c|}
\hline \multirow{2}{*}{ First author, year } & \multirow{2}{*}{ Age } & \multirow{2}{*}{ Male (\%) } & \multirow{2}{*}{$\begin{array}{l}\begin{array}{l}\text { Follow-up } \\
\text { (year) }\end{array} \\
\text { (y) }\end{array}$} & \multirow[b]{2}{*}{ Comparison } & \multicolumn{2}{|c|}{ Treatments and outcomes } & \multirow[b]{2}{*}{ Tumor progression } & \multicolumn{3}{|c|}{ Quality assessment } \\
\hline & & & & & Size & Tumor recurrence & & Random & Blind & Withdraw \\
\hline Mondal, 2016 [43] & $\mathrm{NR}$ & 90 & 0.9 & BCG_SD/MMC & $21 / 19$ & $0 / 2$ & NR & 2 & 1 & 1 \\
\hline Solsona, 2015 [55] & 67 & 89.9 & 7.1 & BCG_MMC/BCG_SD & $211 / 196$ & $44 / 68$ & $26 / 24$ & 2 & $i$ & 1 \\
\hline Inamoto, 2013 [27] & 71.9 & 81.6 & 1.4 & BCG_SD/BCG_LD & $20 / 18$ & $5 / 5$ & $\mathrm{NR}$ & 2 & $i$ & 1 \\
\hline Gontero, 2013 [23] & 67.4 & 85.8 & $\begin{array}{c}1.4 \\
1\end{array}$ & BCG_LD/GEM & $47 / 41$ & $14 / 16$ & $3 / 5$ & 2 & 1 & 1 \\
\hline Oddens, 2013 [45] & 68 & 81.1 & 7.1 & BCG_LD/BCG_SD & $657 / 659$ & $311 / 276$ & $56 / 53$ & 2 & 1 & 1 \\
\hline Järvinen, 2012 [29] & 68 & 80.9 & 7.2 & MMC/BCG_MMC & $40 / 28$ & $35 / 19$ & $14 / 8$ & 2 & 1 & $i$ \\
\hline Gülpınar, 2012 [25] & 58.1 & 80.3 & 5 & BCG_MMC/BCG_SD & $25 / 26$ & $9 / 5$ & $1 / 1$ & 2 & i & 1 \\
\hline Oosterlinck, 2011 [47] & 69 & 86.5 & 4.7 & BCG_MMC/BCG_SD & $48 / 48$ & $23 / 26$ & $2 / 5$ & 2 & i & 1 \\
\hline Hinotsu, 2011 [26] & NR & 90.4 & 2 & BCG_SD/EPI & $83 / 32$ & $19 / 22$ & $3 / 7$ & 2 & $i$ & 1 \\
\hline De Nunzio, 2011 [16] & 60.8 & 68.6 & 7.5 & MMC/Placebo & $97 / 105$ & $10 / 46$ & $0 / 1$ & 2 & i & 1 \\
\hline Bandary, 2011 [11] & 56.2 & NR & 0.9 & GEM/BCG_SD & $40 / 40$ & $10 / 12$ & $\mathrm{NR}$ & 2 & i & 1 \\
\hline O'Brien, $2011[44]$ & 70 & $\mathrm{NR}$ & NR & MMC/Placebo & $105 / 115$ & $21 / 31$ & $\mathrm{NR}$ & 2 & $i$ & 1 \\
\hline Sylvester, 2010 [56] & 67 & NR & 9.2 & EPI/BCG_SD & $267 / 266$ & $147 / 103$ & $24 / 19$ & 2 & i & 1 \\
\hline Porena, 2010 [50] & 69.4 & 84.4 & 3.7 & BCG_SD/GEM & $\begin{array}{l}32 / 32 \\
\end{array}$ & $9 / 17$ & $5 / 10$ & 2 & i & 1 \\
\hline Di Lorenzo, 2010 [18] & 70.4 & 61.2 & 1.3 & GEM/BCG_SD & $40 / 40$ & $21 / 35$ & $7 / 13$ & 2 & 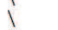 & 1 \\
\hline Addeo, $2010[8]$ & 66.4 & 85.3 & 3 & MMC/GEM & $55 / 54$ & $22 / 15$ & $10 / 6$ & 2 & $i$ & 1 \\
\hline Wu, $2010[63]$ & 65 & 46.9 & 4.6 & EPI/MMC/Placebo & $31 / 27 / 145$ & $9 / 7 / 57$ & $\mathrm{NR}$ & $i$ & 1 & 1 \\
\hline Gudjonsson, 2010 [24] & NR & NR & 2.9 & EPI/Placebo & $102 / 117$ & $63 / 90$ & $\mathrm{NR}$ & 2 & $i$ & 1 \\
\hline Bohle, 2009 [13] & 66 & 79.8 & 2 & GEM/Placebo & $124 / 124$ & $49 / 48$ & $3 / 1$ & 2 & 2 & $i$ \\
\hline Yoneyama, 2008 [64] & 66.7 & 75.3 & 5.6 & BCG_SD/BCG_LD & $85 / 65$ & $29 / 21$ & $\mathrm{NR}$ & $i$ & 1 & 1 \\
\hline Cai, $2008[14]$ & NR & 85.7 & 1.3 & BCG_EPI/BCG_SD & $80 / 81$ & $34 / 40$ & $2 / 4$ & 2 & 2 & $i$ \\
\hline Ojea, 2007 [46] & 64.5 & 87 & 4.4 & BCG_LD/MMC & $281 / 149$ & $88 / 58$ & $32 / 14$ & 2 & 1 & 1 \\
\hline Friedrich, 2007 [22] & 67.4 & 80.2 & 2.9 & BCG_LD/MMC & $163 / 332$ & $41 / 62$ & $\mathrm{NR}$ & 2 & i & 1 \\
\hline El-Ghobashy, 2007 [21] & 62.2 & 80.2 & 2.9 & MMC/Placebo & $31 / 32$ & $12 / 17$ & $1 / 1$ & 2 & & $i$ \\
\hline Di Stasi, 2006 [19] & 67 & 81.6 & 7.3 & BCG_SD/BCG_MMC & $105 / 107$ & $61 / 45$ & $23 / 10$ & 2 & $i$ & 1 \\
\hline de Reijke, 2005 [17] & NR & 92 & 5.6 & BCG_SD/EPI & $84 / 84$ & $9 / 21$ & $\mathrm{NR}$ & 2 & 1 & 1 \\
\hline Cheng, 2005 [15] & 69.9 & 71.2 & 5.1 & BCG_SD/EPI & $102 / 107$ & $30 / 59$ & $9 / 16$ & 2 & i & $i$ \\
\hline Peyromaur, 2004 [49] & 68.4 & 81 & 4.4 & BCG_LD/MMC/Placebo & $57 / 10 / 7$ & $24 / 8 / 2$ & $13 / 1 / 0$ & 1 & i & 1 \\
\hline Irie, 2003 [28] & 61.9 & 85 & 1.7 & BCG_SD/BCG_LD & $39 / 41$ & $5 / 11$ & $2 / 1$ & 1 & & 1 \\
\hline Di Stasi, 2003 [20] & NR & 73 & 3.6 & BCG_SD/MMC & $36 / 72$ & $19 / 46$ & $6 / 14$ & 2 & i & 1 \\
\hline March, 2003 [38] & $\mathrm{NR}$ & $\mathrm{NR}$ & 2.8 & MMC/BCG_LD & $139 / 263$ & $46 / 49$ & $9 / 15$ & 2 & $i$ & $i$ \\
\hline Librenjak, 2003 [35] & 64 & 78.8 & 5.3 & BCG_LD/Placebo & $80 / 90$ & $36 / 68$ & $12 / 28$ & 1 & i & 1 \\
\hline Martinez-Pineiro, 2002 [39] & 63.5 & 90.2 & 5.8 & BCG SD/BCG_LD & $252 / 257$ & $71 / 76$ & $29 / 33$ & $\frac{1}{2}$ & $i$ & 1 \\
\hline Kumar, $2002[33]$ & 56.3 & 88.5 & 2 & BCG_SD/BCG_LD & $13 / 13$ & $4 / 5$ & $1 / 1$ & 2 & & 1 \\
\hline Kolodziej, 2002 [31] & NR & NR & 1.9 & BCG_SD/Placebo & $102 / 53$ & $20 / 29$ & $8 / 12$ & 2 & $i$ & 1 \\
\hline Rajala, 2002 [51] & 65.4 & 72 & 6 & IFN/EP//Placebo & $66 / 68 / 66$ & $45 / 31 / 48$ & $\mathrm{NR}$ & 2 & $i$ & 1 \\
\hline van der Meijden, 2001 [60] & 66 & 77 & 3.5 & EPI/BCG_SD & $279 / 281$ & $142 / 98$ & $19 / 9$ & 2 & i & 1 \\
\hline $\begin{array}{l}\text { Tozawa, } 2001[59] \\
\end{array}$ & 66.3 & 87.5 & 3 & BCG_SD/BCG_EPI & $50 / 23$ & $27 / 13$ & $\mathrm{NR}$ & 1 & i & 1 \\
\hline Bilen, $2000[12]$ & 55 & 95.1 & 1.5 & BCG_SD/BCG_EPI & $21 / 20$ & $4 / 3$ & $2 / 1$ & 2 & & 1 \\
\hline Witjes, 1999 [62] & NR & NR & 2.7 & BCG_MMC/MMC & $90 / 92$ & $39 / 42$ & $5 / 4$ & 2 & i & 1 \\
\hline Malmström, 1999 [37] & $\mathrm{NR}$ & $\mathrm{NR}$ & 5.3 & MMC/BCG_SD & $125 / 125$ & $83 / 66$ & $27 / 20$ & 2 & i & 1 \\
\hline Ali-El-Dein, 1999 [10] & 58.2 & 77.4 & 2.5 & BCG_EPI/BCG_SD & $66 / 58$ & $7 / 12$ & $3 / 5$ & 2 & i & $i$ \\
\hline $\begin{array}{l}\text { Ralaja, } 1999 \text { [52] } \\
\text { [5] }\end{array}$ & $\mathrm{NR}$ & 72 & NR & EPI/IFN/Placebo & $66 / 68 / 66$ & $42 / 23 / 40$ & $\mathrm{NR}$ & 2 & i & 1 \\
\hline Solsona, 1999 [54] & 61 & 92.4 & 7.8 & MMC/Placebo & $57 / 64$ & $23 / 35$ & $1 / 1$ & 2 & & 1 \\
\hline Jimenez-Cruz, 1997 [30] & NR & 84.4 & NR & BCG_SD/IFN & $61 / 49$ & $24 / 34$ & $6 / 7$ & 2 & $i$ & 1 \\
\hline $\begin{array}{l}\text { Ali-el-Dein, } 1997[9] \\
\text { [ }\end{array}$ & 55.7 & 70.8 & 2.7 & EPI/Placebo & $114 / 54$ & $28 / 28$ & $5 / 5$ & 2 & $i$ & 1 \\
\hline Rintala, $1996[53]$ & 68 & 75.5 & 2.8 & MMC/BCG_MMC & $90 / 92$ & $58 / 57$ & $3 / 3$ & 2 & $i$ & 1 \\
\hline Melekos a, $1996[41]$ & NR & NR & 2.9 & $\mathrm{BCG}$ BD /EPI & $46 / 48$ & $16 / 22$ & $5 / 7$ & 2 & 1 & 1 \\
\hline Melekos b, 1996 [42] & $\mathrm{NR}$ & 88.2 & 3.6 & $\mathrm{EPI} / \mathrm{BCG}$ _SD & $61 / 58$ & $34 / 26$ & $10 / 7$ & 2 & i & 1 \\
\hline Krege, $1996[32]$ & NR & 79.8 & 1.7 & BCG_SD/MMC/Placebo & $102 / 112 / 122$ & $26 / 30 / 56$ & $\mathrm{NR}$ & 2 & i & 1 \\
\hline Lundholm, 1996 [36] & 68 & 84 & 3.3 & $\mathrm{MMC} / \mathrm{BCG}_{\text {SD }}$ & $130 / 131$ & $43(125) / 62(125)$ & $17 / 16$ & 2 & $i$ & 1 \\
\hline $\begin{array}{l}\text { Vegt, } 1995[61] \\
\end{array}$ & $\mathrm{NR}$ & NR & 3 & MMC/BCG_SD & $148 / 289$ & $58 / 137$ & $8 / 15$ & 2 & $i$ & 1 \\
\hline Takashi, 1995 [57] & $\begin{array}{l}10 \pi \\
64.7\end{array}$ & $\begin{array}{l}1 \mathrm{NR} \\
83.8\end{array}$ & 2.6 & BCG_SD/BCG_LD & $\begin{array}{l}148 / 289 \\
37 / 37\end{array}$ & $1 / 3$ & $0 / 2$ & 1 & 1 & 1 \\
\hline Lamm, 1995 [34] & 67 & 83 & 2.5 & BCG_LD/MMC & $225 / 222$ & $77 / 101$ & $15 / 25$ & 2 & $i$ & $i$ \\
\hline Oosterlinck, 1993 [48] & NR & NR & 2 & EPI/Placebo & $194 / 205$ & $41 / 49$ & $7 / 5$ & 2 & $i$ & 1 \\
\hline Melekos, $1993[40]$ & NR & 83.2 & $\mathrm{NR}$ & EPI/BCG_SD/Placebo & $67 / 62 / 32$ & $27 / 20 / 19$ & $6 / 4 / 7$ & 2 & $i$ & 1 \\
\hline Tolley, 1988 [58] & NR & NR & 1 & MMC/Placebo & $267 / 130$ & $\begin{array}{l}137 / 84 \\
\end{array}$ & $\mathrm{NR}$ & 2 & 1 & 1 \\
\hline
\end{tabular}

Fig. 2. Network geometry of clinical trials of postoperative intravesical instillation for efficacy in bladder cancer. * BCG_SD: Bacille Calmette Guerin standard dose; BCG_LD: Bacille Calmette Guerin low dose; MMC: Mitomycin C; GEM: Gemcitabine; EPI: Epirubicin; IFN: Interferon.
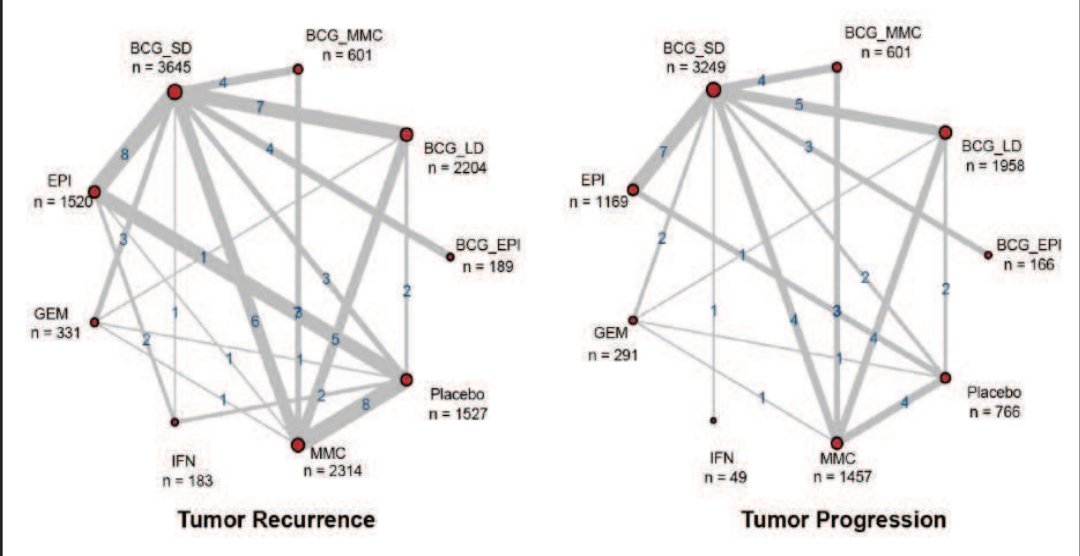

1.30-1.82). Postoperative BC patients with EPI also appeared to have a higher risk of tumor progression compared to those with BCG_SD $(O R=1.64,95 \%$ CI $=1.16-2.33)$. As suggested by pairwise meta-analysis, BCG_SD was the only one that was likely to inhibit the risk of tumor progression $(\mathrm{OR}=0.33,95 \% \mathrm{CI}=0.15-0.71)$. No significant study heterogeneity was identified for the above results. 


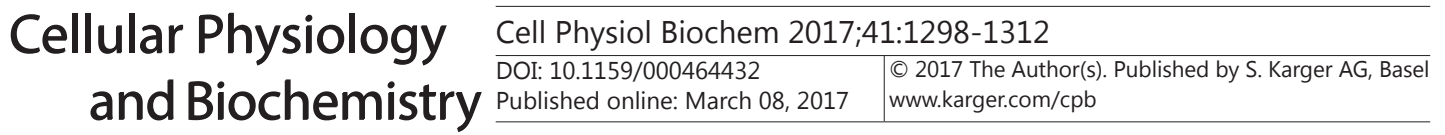

Wu et al.: Assessment of Therapies in Bladder Cancer

\begin{tabular}{|c|c|c|c|c|c|c|}
\hline Table & Endpoints & Direct comparisons & $I^{2}$ & $P_{H}$ values & OR $(95 \% \mathrm{CI})$ & $P_{O R}$ values \\
\hline Pair-wise me- & \multirow[t]{19}{*}{ Tumor Recurrence } & GEM vs. BCG_LD & - & - & $1.31(0.57,3.01)$ & 0.524 \\
\hline & & MMC vs. BCG_LD & $53.10 \%$ & 0.074 & $1.26(0.93,1.70)$ & 0.141 \\
\hline ta-analyses of & & BCG+EPI vs. BCG_SD & $0.00 \%$ & 0.748 & $0.82(0.55,1.23)$ & 0.347 \\
\hline direct & & BCG_LD vs. BCG_SD & $0.00 \%$ & 0.895 & $1.12(0.95,1.32)$ & 0.164 \\
\hline 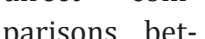 & & BCG+MMC vs. BCG_SD & $11.10 \%$ & 0.338 & $0.74(0.54,0.99)$ & 0.049 \\
\hline parisons det & & EPI vs. BCG_SD & $0.00 \%$ & 0.537 & $1.53(1.30,1.82)$ & $<0.001$ \\
\hline ween different & & GEM vs. BCG_SD & $45.70 \%$ & 0.158 & $0.93(0.48,1.82)$ & 0.831 \\
\hline treatments. * & & IFN vs. BCG_SD & - & - & $1.76(0.93,3.36)$ & 0.084 \\
\hline & & MMC vs. BCG_SD & $14.70 \%$ & 0.32 & $0.97(0.77,1.22)$ & 0.767 \\
\hline$P_{\mathrm{H}} P$ value of & & IFN vs. EPI & $83.00 \%$ & 0.015 & $0.90(0.33,2.47)$ & 0.834 \\
\hline heterogenei- & & $B C G+M M C$ vs. MMC & $0.00 \%$ & 0.881 & $0.92(0.67,1.26)$ & 0.605 \\
\hline ty; OR: Odds & & EPI vs. MMC & - & - & $1.12(0.37,3.41)$ & 0.842 \\
\hline & & GEM vs. MMC & - & - & $0.69(0.33,1.48)$ & 0.345 \\
\hline Io; Cl: Con- & & BCG_LD vs. Placebo & $6.40 \%$ & 0.301 & $0.66(0.38,1.16)$ & 0.146 \\
\hline fidential inter- & & BCG_SD vs. Placebo & $0.00 \%$ & 0.565 & $0.48(0.34,0.70)$ & $<0.001$ \\
\hline & & EPI vs. Placebo & $0.00 \%$ & 0.594 & $0.76(0.62,0.94)$ & 0.01 \\
\hline D. & & GEM vs. Placebo & - & - & $1.02(0.64,1.63)$ & 0.931 \\
\hline Bacille & & IFN vs. Placebo & $36.20 \%$ & 0.211 & $0.74(0.45,1.23)$ & 0.246 \\
\hline mette & & MMC vs. Placebo & $39.30 \%$ & 0.117 & $0.65(0.48,0.88)$ & 0.005 \\
\hline standard & \multirow[t]{16}{*}{ Tumor Progression } & GEM vs. BCG_LD & - & - & $1.91(0.43,8.49)$ & 0.395 \\
\hline rin standard & & MMC vs. BCG_LD & $0.80 \%$ & 0.388 & $1.13(0.75,1.69)$ & 0.566 \\
\hline dose; BCG_LD: & & BCG+MMC vs. BCG_SD & $13.40 \%$ & 0.326 & $0.69(0.41,1.16)$ & 0.163 \\
\hline Bacille Cal- & & BCG_LD vs. BCG_SD & $0.00 \%$ & 0.951 & $1.08(0.79,1.46)$ & 0.645 \\
\hline Dachine st & & EPI vs. BCG_SD & $0.00 \%$ & 0.587 & $1.64(1.16,2.33)$ & 0.006 \\
\hline & & GEM vs. BCG_SD & $63.30 \%$ & 0.099 & $1.00(0.28,3.62)$ & 0.998 \\
\hline rin low dose; & & BCG+EPI vs. BCG_SD & $0.00 \%$ & 0.999 & $0.52(0.19,1.44)$ & 0.209 \\
\hline & & MMC vs. BCG_SD & $0.00 \%$ & 0.956 & $1.18(0.80,1.74)$ & 0.41 \\
\hline & & IFN vs. BCG_SD & - & - & $1.45(0.46,4.60)$ & 0.526 \\
\hline cin C; GEM: & & BCG+MMC vs. MMC & $0.00 \%$ & 0.871 & $0.96(0.47,1.97)$ & 0.912 \\
\hline Gemcitabine; & & GEM vs. MMC & - & - & $0.61(0.21,1.80)$ & 0.371 \\
\hline , & & MMC vs. Placebo & $0.00 \%$ & 0.998 & $1.01(0.25,4.14)$ & 0.993 \\
\hline & & GEM vs. Placebo & - & - & $3.00(0.31,29.24)$ & 0.344 \\
\hline cin; IFN: Inter- & & BCG_LD vs. Placebo & $23.10 \%$ & 0.254 & $0.63(0.22,1.77)$ & 0.375 \\
\hline & & BCG_SD vs. Placebo & $0.00 \%$ & 0.845 & $0.33(0.15,0.71)$ & 0.004 \\
\hline & & EPI vs. Placebo & $27.00 \%$ & 0.254 & $0.67(0.30,1.51)$ & 0.335 \\
\hline
\end{tabular}

Table 3. The efficacy of different treatments according to the network meta-analysis using ORs and corresponding 95\% CrIs. * OR: Odds ratio; CrI: Credible interval; BCG_SD: Bacille Calmette Guerin standard dose; BCG_LD: Bacille Calmette Guerin low dose; MMC: Mitomycin C; GEM: Gemcitabine; EPI: Epirubicin; IFN: Interferon

\begin{tabular}{|c|c|c|c|c|c|c|c|c|}
\hline \multicolumn{9}{|l|}{ Tumor Recurrence } \\
\hline BCG_EPI & $1.48(0.68,3.29)$ & $1.11(0.47,2.59)$ & $1.38(0.68,2.77)$ & $2.75(1.26,5.99)$ & $1.62(0.66,3.90)$ & $2.77(1.05,7.32)$ & $1.84(0.87,4.01)$ & $4.18(1.92,9.21)$ \\
\hline $0.68(0.30,1.48)$ & BCG_LD & $0.75(0.42,1.31)$ & $0.93(0.64,1.34)$ & $1.86(1.16,2.97)$ & $1.09(0.59,1.97)$ & $1.88(0.89,3.94)$ & $1.26(0.87,1.82)$ & $2.83(1.84,4.35)$ \\
\hline $0.90(0.39,2.12)$ & $1.34(0.76,2.36)$ & BCG_MMC & $1.25(0.76,2.01)$ & $2.51(1.40,4.44)$ & $1.48(0.72,2.94)$ & $2.51(1.12,5.64)$ & $1.68(1.02,2.77)$ & $3.82(2.18,6.62)$ \\
\hline $0.73(0.36,1.46)$ & $1.07(0.75,1.57)$ & $0.80(0.50,1.31)$ & BCG_SD & $2.01(1.42,2.83)$ & $1.17(0.68,2.03)$ & $2.01(1.03,3.97)$ & $1.35(0.98,1.88)$ & $3.06(2.16,4.35)$ \\
\hline $0.36(0.17,0.79)$ & $0.54(0.34,0.86)$ & $0.40(0.23,0.71)$ & $0.50(0.35,0.70)$ & EPI & $0.59(0.32,1.07)$ & $1.01(0.52,1.95)$ & $0.67(0.44,1.03)$ & $1.52(1.05,2.20)$ \\
\hline $0.62(0.26$ & $0.91(0.51,1.70)$ & $0.68(0.34,1.39)$ & $0.85(0.49,1.48)$ & $1.70(0.93,3.16)$ & GEM & $1.72(0.74,4.01)$ & $1.14(0.65,2.05)$ & $2.59(1.46,4.66)$ \\
\hline $0.36(0.14,0.95)$ & $0.53(0.25,1.13)$ & $0.40(0.18,0.90)$ & $0.50(0.25,0.97)$ & $0.99(0.51,1.93)$ & $0.58(0.25,1.35)$ & IFN & $0.67(0.33,1.36)$ & $1.51(0.77,2.94)$ \\
\hline $0.54(0.25,1.15)$ & $0.79(0.55,1.15)$ & $0.59(0.36,0.98)$ & $0.74(0.53,1.02)$ & $1.49(0.97,2.25)$ & $0.88(0.49,1.54)$ & $1.49(0.73,3.03)$ & MMC & $2.27(1.58,3.19)$ \\
\hline $0.24(0.11,0.52)$ & MMC vs. BCG_SD & $0.26(0.15,0.46)$ & $0.33(0.23,0.46)$ & $0.66(0.45,0.95)$ & $0.39(0.21,0.68)$ & $0.66(0.34,1.30)$ & $0.44(0.31,0.63)$ & Placebo \\
\hline \multicolumn{9}{|l|}{ Tumor Progression } \\
\hline BCG_EPI & $2.2(0.76,7.54)$ & $1.57(0.49,5.53)$ & $2.05(0.73,6.69)$ & $3.63(1.22,12.3)$ & $2.32(0.7,8.94)$ & $3.22(0.66,17.64)$ & $2.46(0.84,8.41)$ & $4.62(1.51,16.6)$ \\
\hline $0.45(0.13,1.31)$ & BCG_LD & $0.69(0.41,1.21)$ & $0.93(0.66,1.26)$ & $1.63(1.00,2.56)$ & $1.04(0.56,1.99)$ & $1.46(0.41,5.26)$ & $1.11(0.77,1.55)$ & $2.08(1.25,3.49)$ \\
\hline $0.64(0.18,2.03)$ & $1.45(0.83,2.44)$ & BCG_MMC & $1.32(0.85,2.05)$ & $2.34(1.31,4.10)$ & $1.49(0.76,3.29)$ & $2.10(0.56,8.00)$ & $1.60(0.89,2.59)$ & $3.00(1.57,5.87)$ \\
\hline $0.49(0.15,1.38)$ & $1.07(0.79,1.52)$ & $0.76(0.49,1.17)$ & BCG_SD & $1.77(1.22,2.53)$ & $1.14(0.61,2.10)$ & $1.58(0.47,5.58)$ & $1.19(0.87,1.67)$ & $2.25(1.39,3.67)$ \\
\hline $0.28(0.08,0.82)$ & $0.61(0.39,0.99)$ & $0.43(0.24,0.76)$ & $0.57(0.39,0.82)$ & EPI & $0.64(0.31,1.31)$ & $0.90(0.25,3.25)$ & $0.68(0.42,1.11)$ & $1.28(0.76,2.23)$ \\
\hline $0.43(0.11,1.42)$ & $0.96(0.50,1.79)$ & $0.67(0.30,1.32)$ & $0.88(0.48,1.63)$ & $1.57(0.76,3.22)$ & GEM & $1.40(0.35,5.37)$ & $1.06(0.55,1.93)$ & $2.01(0.95,4.06)$ \\
\hline $0.31(0.06,1.52)$ & $0.68(0.19,2.46)$ & $0.48(0.12,1.79)$ & $0.63(0.18,2.14)$ & $1.11(0.31,3.97)$ & $0.71(0.19,2.89)$ & IFN & $0.75(0.21,2.75)$ & $1.43(0.38,5.31)$ \\
\hline $0.41(0.12,1.20)$ & $0.90(0.64,1.30)$ & $0.63(0.39,1.13)$ & $0.84(0.60,1.15)$ & $1.48(0.90,2.36)$ & $0.94(0.52,1.80)$ & $1.34(0.36,4.76)$ & MMC & $1.88(1.09,3.35)$ \\
\hline $0.22(0.06,0.66)$ & $0.48(0.29,0.80)$ & $0.33(0.17,0.64)$ & $0.44(0.27,0.72)$ & $0.78(0.45,1.32)$ & $0.50(0.25,1.05)$ & $0.70(0.19,2.66)$ & $0.53(0.30,0.91)$ & Placebo \\
\hline
\end{tabular}

Network meta-analysis

The efficacy of different intravesical instillation therapies was also assessed by using indirect evidence. Patients with seven intravesical instillation therapies benefited from a 


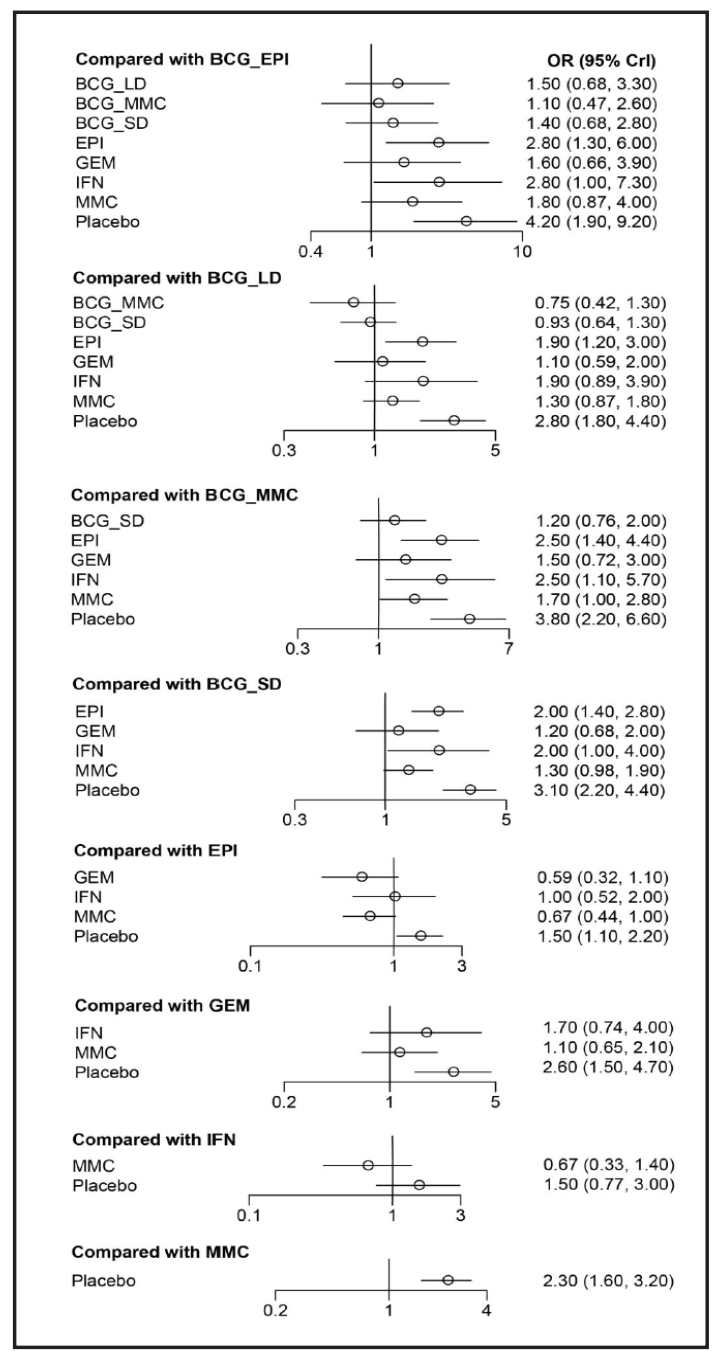

Fig. 3. Odds ratio and $95 \%$ credible interval for tumor recurrence. ${ }^{*}$ OR: Odds ratio; CrI: Credible interval; BCG_SD: Bacille Calmette Guerin standard dose; BCG_LD: Bacille Calmette Guerin low dose; MMC: Mitomycin C; GEM: Gemcitabine; EPI: Epirubicin; IFN: Interferon.

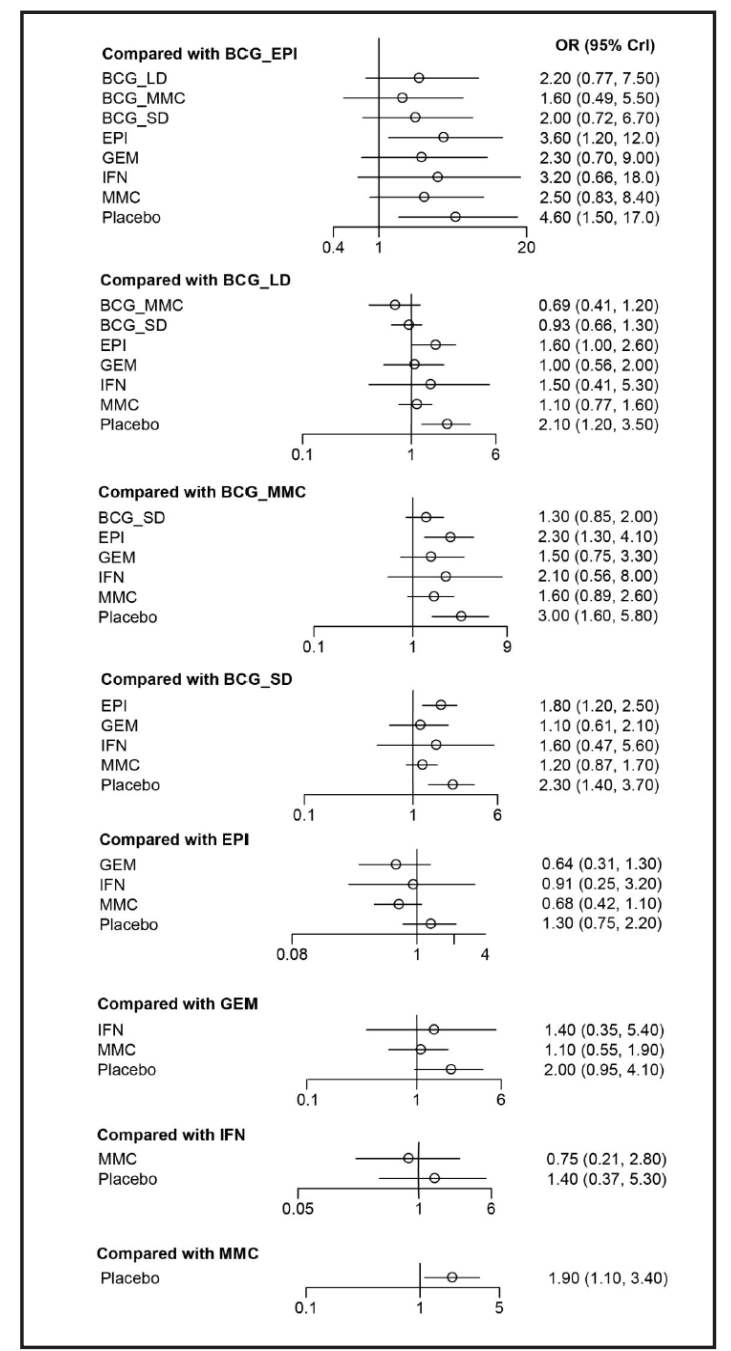

Fig. 4. Odds ratio and 95\% credible interval for tumor progression. * OR: Odds ratio; CrI: Credible interval; BCG_SD: Bacille Calmette Guerin standard dose; BCG_LD: Bacille Calmette Guerin low dose; MMC: Mitomycin C; GEM: Gemcitabine; EPI: Epirubicin; IFN: Interferon.

reduced risk of tumor recurrence compared to those with placebo (BCG_EPI, BCG_LD, BCG_ MMC, BCG_SD, EPI, GEM and MMC) (all ORs < 1, Table 3, Fig. 3). Patients with BCG_EPI, BCG_ LD, BCG_MMC and BCG_SD appeared to have a decreased risk of tumor recurrence compared to those with EPI (all ORs $<1$ ). Also, three intravesical instillation therapies were associated with a suppressed risk of tumor recurrence compared to IFN: BCG_EPI, BCG_MMC and BCG_ SD (all ORs < 1). Patients with BCG_EPI, BCG_LD, BCG_MMC and BCG_SD were associated with a reduced risk of tumor progression compared to EPI (all ORs $<1$, Table 3, Fig. 4). Besides that, five intravesical instillation therapies (BCG_EPI, BCG_LD, BCG_MMC, BCG_SD, and $\mathrm{MMC}$ ) were likely to inhibit the risk of tumor progression (all ORs $<1$ ). The cumulative probability of being a specific rank was displayed in Fig. 5. BCG_EPI, BCG_MMC and BCG_SD exhibited the three highest SUCRA values in regards of tumor recurrence (BCG_EPI $=0.88$, BCG_MMC $=0.86$, BCG_SD $=0.71$ ) and tumor progression $\left(B C G \_E P I=0.92, B C G \_M M C=0.84\right.$, BCG_SD $=0.65$ ). 


\section{Cellular Physiology Cell Physiol Biochem 2017;41:1298-1312 and Biochemistry DOI: 10.1159/000464432 2017 O 2017 The Author(s). Published by S. Karger AG, Basel

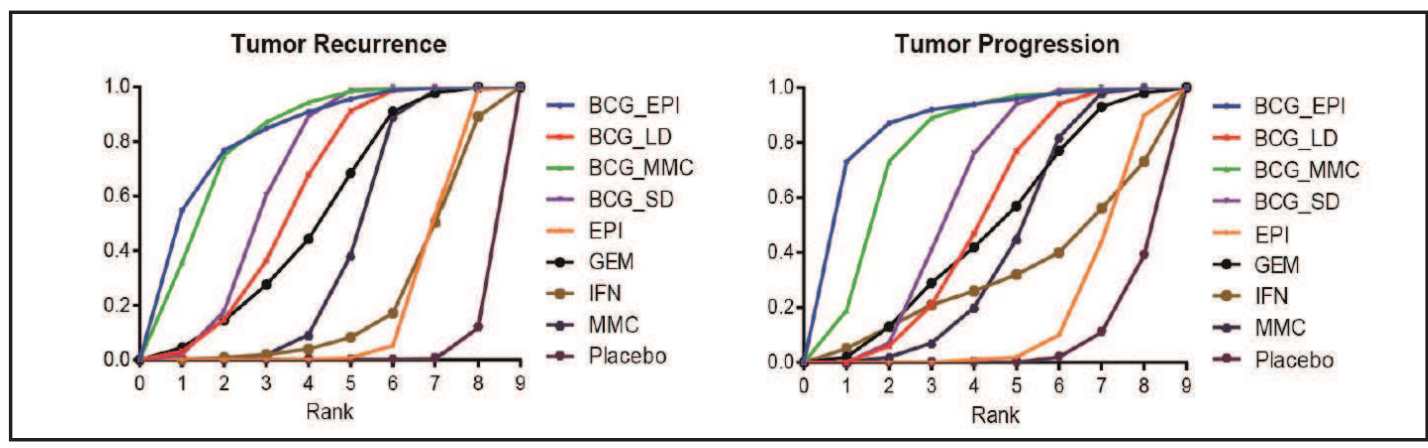

Fig. 5. Ranking of treatments in terms of different criteria. * BCG_SD: Bacille Calmette Guerin standard dose; BCG_LD: Bacille Calmette Guerin low dose; MMC: Mitomycin C; GEM: Gemcitabine; EPI: Epirubicin; IFN: Interferon.

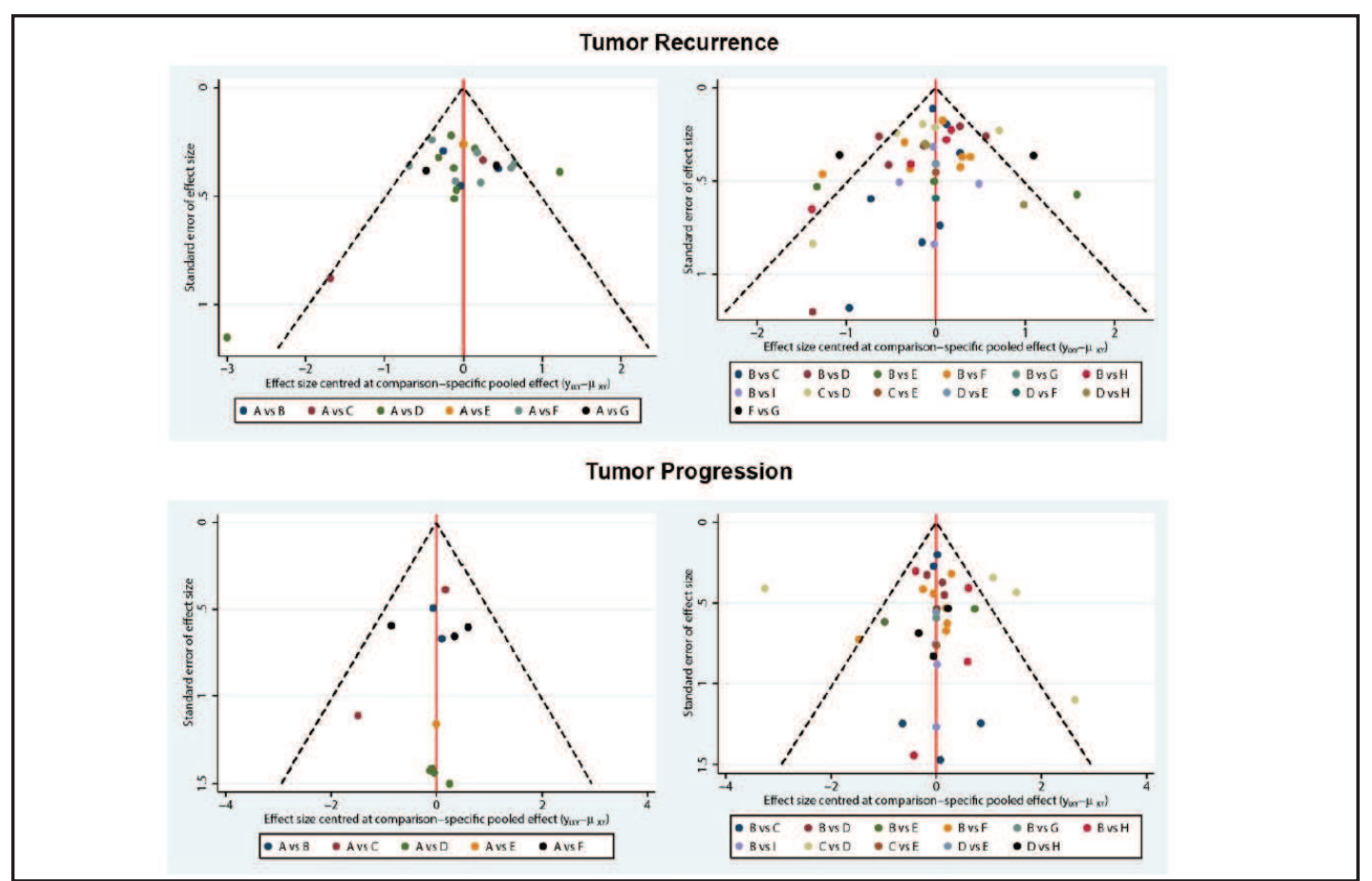

Fig. 6. Comparison adjusted funnel plot for assessing publication bias. * A: Placebo; B: Bacille Calmette Guerin standard dose; C: Bacille Calmette Guerin low dose; D: Mitomycin C; E: Gemcitabine; F: Epirubicin; G: Interferon; H: Bacille Calmette Guerin/ Mitomycin C; I: Bacille Calmette Guerin/ Epirubicin.

\section{Model assumption assessments}

Results of the node splitting method are depicted in Table 4. The consistency assumption in the NMA and MCMC simulation was not violated since inconsistency in the corresponding evidence was not significant (all $P$-value $>0.05$ ). Besides that, small-study effects and publication bias was visually examined by funnel plots (Fig. 6). Since no asymmetry patterns were observed in the funnel plots, we concluded that there was no strong evidence of publication bias.

\section{Discussion}

Overall, some consistent evidence was provided by our pairwise MA and NMA. A reduced risk of tumor recurrence was observed in postoperative patients with BCG_SD, EPI or MMC. Besides that, both MA and NMA confirmed that BCG_SD was more effective than EPI 


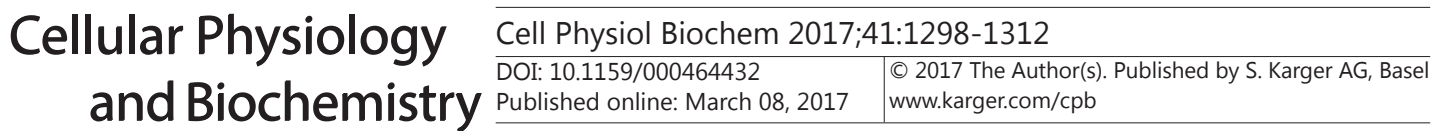

Wu et al.: Assessment of Therapies in Bladder Cancer

Table 4. Comparison of direct and indirect evidences. * BCG_SD: Bacille Calmette Guerin standard dose; BCG_LD: Bacille Calmette Guerin low dose; MMC: Mitomycin C; GEM: Gemcitabine; EPI: Epirubicin; IFN: Interferon

\begin{tabular}{|c|c|c|c|c|c|c|c|}
\hline Endpoints & Comparisons & OR ( $95 \%$ CI or CrI) & $P$ values & Endpoints & Comparisons & OR $(95 \%$ CI or CrI ) & $P$ values \\
\hline Tumor Recurrence & BCG_SD vs BCG_LD & & \multirow{4}{*}{0.310} & & direct & $1.90(0.90,4.70)$ & \multirow{4}{*}{0.220} \\
\hline & direct & $0.79(0.46,1.30)$ & & & indirect & $0.79(0.24,2.80)$ & \\
\hline & indirect & $1.10(0.68,1.90)$ & & & network & $1.50(0.79,3.00)$ & \\
\hline & network & $0.93(0.63,1.30)$ & & & Placebo vs MMC & & \\
\hline & GEM vs BCG_LD & & \multirow{4}{*}{0.583} & & direct & $2.00(1.20,3.20)$ & \multirow{3}{*}{0.320} \\
\hline & direct & $1.50(0.40,5.90)$ & & & indirect & $2.90(1.60,5.10)$ & \\
\hline & indirect & $0.99(0.47,2.00)$ & & & network & $2.30(1.60,3.10)$ & \\
\hline & network & $1.10(0.58,2.00)$ & & Tumor Progression & BCG_SD vs BCG_LD & & \multirow{5}{*}{0.891} \\
\hline & MMC vs BCG_LD & & \multirow{4}{*}{0.376} & & direct & $0.91(0.60,1.30)$ & \\
\hline & direct & $1.50(0.92,2.60)$ & & & indirect & $0.97(0.58,1.60)$ & \\
\hline & indirect & $1.10(0.64,1.90)$ & & & network & $0.91(0.67,1.30)$ & \\
\hline & network & $1.30(0.86,1.80)$ & & & GEM vs BCG_LD & & \\
\hline & Placebo vs BCG_LD & & \multirow{4}{*}{0.744} & & direct & $2.10(0.45,11.0)$ & \multirow{4}{*}{0.395} \\
\hline & direct & $2.30(0.78,5.90)$ & & & indirect & $0.94(0.45,2.00)$ & \\
\hline & indirect & $2.80(1.80,4.50)$ & & & network & $1.00(0.56,1.90)$ & \\
\hline & network & $2.80(1.80,4.30)$ & & & MMC vs BCG_LD & & \\
\hline & BCG_SD vs BCG_MMC & & \multirow{4}{*}{0.441} & & direct & $1.10(0.69,1.70)$ & \multirow{4}{*}{0.945} \\
\hline & direct & $1.50(0.79,2.60)$ & & & indirect & $1.10(0.67,1.80)$ & \\
\hline & indirect & $0.97(0.44,2.20)$ & & & network & $1.10(0.78,1.60)$ & \\
\hline & network & $1.30(0.76,2.00)$ & & & Placebo vs BCG_LD & & \\
\hline & MMC vs BCG_MMC & & \multirow{4}{*}{0.516} & & direct & $1.80(0.79,3.80)$ & \multirow{4}{*}{0.560} \\
\hline & direct & $1.40(0.68,3.10)$ & & & indirect & $2.40(1.20,4.80)$ & \\
\hline & indirect & $2.00(1.00,4.10)$ & & & network & $2.10(1.30,3.40)$ & \\
\hline & network & $1.70(1.00,2.80)$ & & & BCG_SD vs BCG_MMC & & \\
\hline & EPI vs BCG_SD & & \multirow{4}{*}{0.349} & & direct & $1.60(0.94,2.70)$ & \\
\hline & direct & $2.30(1.50,3.40)$ & & & indirect & $0.81(0.33,1.90)$ & 0.219 \\
\hline & indirect & $1.60(0.88,3.00)$ & & & network & $1.30(0.86,2.00)$ & \\
\hline & network & $2.00(1.40,2.80)$ & & & MMC vs BCG_MMC & & \\
\hline & GEM vs BCG SD & & & & direct & $1.00(0.50,2.30)$ & \\
\hline & direct & $0.70(0.32,1.70)$ & & & indirect & $2.00(1.10,3.90)$ & 0.180 \\
\hline & indirect & $1.80(0.82,3.70)$ & 0.124 & & network & $1.60(0.99,2.60)$ & \\
\hline & network & $1.20(0.68,2.00)$ & & & EPI vs BCG_SD & & \\
\hline & IFN vs BCG_SD & $1.20[0.00,2.00]$ & & & direct & $1.80(1.20,2.70)$ & \\
\hline & direct & $3.60(1.00,13.00)$ & & & indirect & $1.80(0.55,5.80)$ & 0.994 \\
\hline & indirect & $1.60(0.71,3.50)$ & 0.280 & & network & $1.70(1.20,2.50)$ & \\
\hline & network & $2.00(1.00,3.90)$ & 0.280 & & GEM vs BCG_SD & & \\
\hline & MMC vs BCG SD & & & & direct & $0.94(0.41,2.20)$ & \\
\hline & direct & $1.10(0.65,1.80)$ & & & indirect & $1.40(0.63,3.50)$ & 0.488 \\
\hline & indirect & $1.60(1.10,2.50)$ & 0.191 & & network & $1.10(0.63,2.10)$ & \\
\hline & network & $1.40(0.98,1.90)$ & & & MMC vs BCG_SD & & \\
\hline & Placebo vs BCG_SD & $1.40(0.98,1.90)$ & & & direct & $1.20(0.74,1.90)$ & \\
\hline & direct & $3.40(1.70,7.30)$ & & & indirect & $1.20(0.76,1.90)$ & 0.999 \\
\hline & indirect & $3.00(2.00,4.60)$ & 0.766 & & network & $1.20(0.88,1.70)$ & \\
\hline & network & $3.10(2.20,4.40)$ & & & Placebo vs BCG_SD & & \\
\hline & $\begin{array}{l}\text { network } \\
\text { IFN vs EPI }\end{array}$ & $3.10(2.20,4.40)$ & & & direct & $3.80(1.70,9.20)$ & \\
\hline & direct & $0.86(0.37,1.90)$ & & & indirect & $1.70(0.92,3.00)$ & 0.111 \\
\hline & indirect & $1.90(0.47,6.20)$ & 0.339 & & network & $2.30(1.40,3.70)$ & \\
\hline & network & $0.99(0.51,1.90)$ & & & Placebo vs EPI & & \\
\hline & MMC vs EPI & & & & direct & $1.60(0.74,3.50)$ & \\
\hline & $\begin{array}{l}\text { direct } \\
\text { dill EST }\end{array}$ & $0.83(0.18,4.20)$ & & & indirect & $1.10(0.49,2.30)$ & 0.534 \\
\hline & indirect & $0.66(0.43,1.00)$ & 0.809 & & network & $1.30(0.80,2.20)$ & \\
\hline & $\begin{array}{l}\text { Indirect } \\
\text { network }\end{array}$ & $0.67(0.45,1.10)$ & 0.809 & & MMC vs GEM & & \\
\hline & Placebo vs EPI & $0.07(0.4 .5,1.10)$ & & & direct & $1.80(0.52,6.30)$ & \\
\hline & direct & $1.90(1.20,2.90)$ & & & indirect & $0.83(0.36,1.70)$ & 0.266 \\
\hline & $\begin{array}{l}\text { anrect } \\
\text { indirect }\end{array}$ & $1.00(0.55,1.90)$ & 0.128 & & network & $1.10(0.56,2.00)$ & \\
\hline & network & $1.50(1.10,2.20)$ & & & Placebo vs GEM & & \\
\hline & MMC vs GEM & & & & direct & $0.29(0.01,4.70)$ & \\
\hline & $\begin{array}{l}\text { MIMC VS GEM } \\
\text { direct }\end{array}$ & $1.70(0.49,6.10)$ & & & indirect & $2.50(1.20,5.40)$ & 0.119 \\
\hline & indirect & $1.00(0.55,2.00)$ & 0.474 & & network & $2.10(0.97,4.10)$ & \\
\hline & network & $1.10(0.66,2.10)$ & 0.474 & & Placebo vs MMC & & \\
\hline & Placebo vs GEM & & & & direct & $1.00(0.18,6.90)$ & \\
\hline & $\begin{array}{l}\text { Placebo vs GEM } \\
\text { direct }\end{array}$ & $0.95(0.34,2.80)$ & & & indirect & $2.20(1.20,4.00)$ & 0.403 \\
\hline & indirect & $3.80(2.00,7.50)$ & 0.024 & & network & $1.90(1.10,3.20)$ & \\
\hline & network & $2.60(1.50,4.70)$ & & & & & \\
\hline
\end{tabular}

considering the risk of tumor recurrence and progression. On the other hand, BCG_SD was the only intravesical instillation agent which may suppress the risk of tumor progression in postoperative BC patients. Although results provided by NMA are more informative than those provided by MA, ranking various intravesical instillation agents remains challenging. Results of SUCRA indicate that BCG_EPI, BCG_MMC and BCG_SD outperformed others with respect to tumor recurrence and progression.

The bladder is able to trigger strong immune responses and animal models indicated that the effect of BCG was maximized if there was close contact between BCG and cancer $[65,66]$. BCG immunotherapy has not been widely accepted for BC patients until the study which was conducted by the Southwest Oncology Group in 1980 [67]. The corresponding 


\section{Cellular Physiology Cell Physiol Biochem 2017;41:1298-1312

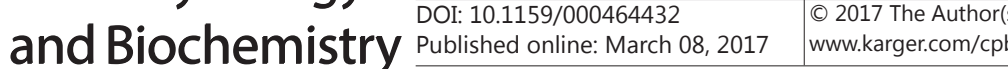

Wu et al.: Assessment of Therapies in Bladder Cancer

results revealed that BC patients with BCG immunoprophylaxis after local surgery exhibited decreased tumor recurrence rate and increased median time to recurrence. Results of our study appear to support the above conclusions since post-surgery patients with BCG SD exhibited lower risk of tumor recurrence and progression than those with placebo. This convinces us that BCG has specific efficacy for preventing tumor recurrence and progression in $\mathrm{BC}$ patients. On the other hand, intravesical chemotherapy prophylaxis for tumor recurrence, progression or mortality did not exhibit strong efficacy in the long term [4]. This disadvantage may be overcome by BCG immunotherapy since the average shortterm reduction in tumor recurrence among BC patients treated by BCG is about $40 \%$ and the corresponding benefits can persist for 5 to 10 years [68]. Clinical trials have compared BCG immunotherapy with other forms of intravesical chemotherapies. In our study, BCG_SD was compared with EPI, GEM and MMC. However, pooled evidence demonstrated that only patients treated by BCG_SD exhibited a lower risk of tumor recurrence and progression than those with EPI. Therefore, the dispute of whether BCG immunotherapy is more efficacious than intravesical chemotherapies in postoperative BC patients should be studied further. Despite the convincible performance of BCG_SD in our study, using BCG immunotherapy for $\mathrm{BC}$ patients has some inevitable drawbacks. First, predicting whether a specific $\mathrm{BC}$ patient will respond to BCG immunotherapy cannot be achieved since no significant prognostic factors have been discovered [68]. As a result, clinicians will not be able to know in advance whether BCG immunotherapy can provide adequate efficacy given a specific BC patient. Several issues may come after this, for instance, if clinicians believed that BCG the optimal option for preventing tumor recurrence and progression based on empirical evidence, abuse of BCG immunotherapy and waste of resources may occur in BC patients with different tumor classifications or stages. It has been verified that BCG is not appropriate for muscle-invasive $\mathrm{BC}$ or for tumors that lie deep within the prostate or in the upper urinary tract [1].Thus, the selection of an appropriate intravesical instillation therapy should be carefully planned after pathological classification and tumor staging.

Another major issue associated with BCG immunotherapy is its side effects such as cystitis, dysuria, occasional haematuria and BCG sepsis [1]. Recently, studies investigating the feasibility of BCG dose reduction appeared to flourish in the current literature. These studies suggest that standard dose of BCG immunotherapy can be halved in most circumstances, resulting in a reduction in side effects without any significant loss of efficacy [1]. The above hypothesis was also tested and partially verified by our analysis in which BCG immunotherapy with standard dose was compared to that with low dose. In our study, BC patients with BCG SD or BCG_LD had the same level of tumor recurrence/progression risk. The SUCRA value of BCG_LD is marginally lower than that of BCG_SD, suggesting the two may have approximately the same level of efficacy for preventing tumor recurrence and progression. Evidence also indicated that a three-fold decrease in the dose of BCG immunotherapy is as effective as the standard dose against the risk of tumor progression but with significantly less toxicity [69]. According to the above evidence, we suspected that the relationship between the dose of BCG immunotherapy and BC patients' responses to BCG is not linear; therefore reducing the dose of BCG immunotherapy is likely to maintain and even enhance responses in vivo. However, the above hypothesis together with the reduction in toxicity was not verified by our study and should be confirmed by ongoing researches.

Our study also investigated the potential benefits of alternating or sequentially combined intravesical instillation therapies in $\mathrm{BC}$ patients. This strategy has three potential advantages: reducing the use of intravesical BCG, reducing the toxicity resulted from intravesical BCG and enhancing the corresponding efficacy. Two alternating or sequentially combined intravesical instillation therapies were included and compared with other standard approaches: BCG_ MMC and BCG_EPI. Both pairwise MA and NMA suggest no significant difference in the risk of tumor recurrence or progression between patients with BCG_EPI and BCG_SD. This finding was verified by an individual study which suggested that the combination therapy of BCG_EPI should not be recommended to high risk patients with superficial BC [70]. Instead, this combination therapy led to a higher incidence of side-effects without any additional 


\section{Cellular Physiology Cell Physiol Biochem 2017;41:1298-1312

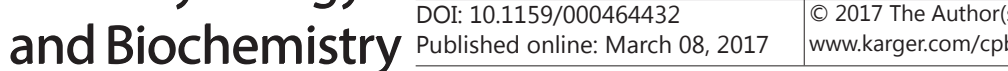

Wu et al.: Assessment of Therapies in Bladder Cancer

benefits compared to standard BCG. A similar trend between patients with BCG_MMC and those with BCG_SD was suggested by our analysis. Although BCG_EPI or BCG_MMC exhibited a larger SUCRA value than BCG_SD, it is still unclear whether alternating or sequentially combined instillation therapies are more efficacious than BCG.

Our study enhanced the level of evidence by incorporating a large number of studies and patients. Nevertheless, heterogeneity of included studies may impact on the above conclusions. For instance, variation in patient age, follow-up duration, dose and particularly the classification of $\mathrm{BC}$ in each study reflects a high degree of heterogeneity which cannot be addressed in the process of evidence. Besides that, we did not include any unpublished articles or studies and this may result in potential biased results. Thirdly, the proportion of male $\mathrm{BC}$ patients is substantially larger than that of female BC patients in each included study. If gender is a significant risk factor for tumor recurrence or progression, then unbalanced distribution of male or females in the study may cause biased results. In conclusion, BCG SD, EPI and MMC appeared to have established efficacy for preventing tumor recurrence in postoperative BC patients. The efficacy of BCG may not be significantly reduced if standard dose was reduced to a lower level. However, we may experience pressing challenges in the future when increasing BC patients accompanied with shortage of intravesical BCG continue since there is no unanimous agreement suggesting that standard intravesical BCG can be replaced by alternating or sequentially combined intravesical instillation therapies.

\section{Disclosure Statement}

The authors declare no conflict of interest.

\section{References}

$>1$ Alexandroff AB, Jackson AM, O'Donnell MA, James K: BCG immunotherapy of bladder cancer: 20 years on. Lancet 1999;353:1689-1694.

2 Mostafid AH, Palou Redorta J, Sylvester R, Witjes JA: Therapeutic options in high-risk non-muscle-invasive bladder cancer during the current worldwide shortage of bacille Calmette-Guerin. Eur Urol 2015;67:359360.

-3 Pawinski A, Sylvester R, Kurth KH, Bouffioux C, van der Meijden A, Parmar MK, Bijnens L: A combined analysis of European Organization for Research and Treatment of Cancer, and Medical Research Council randomized clinical trials for the prophylactic treatment of stage TaT1 bladder cancer. European Organization for Research and Treatment of Cancer Genitourinary Tract Cancer Cooperative Group and the Medical Research Council Working Party on Superficial Bladder Cancer. J Urol 1996;156:1934-1940, discussion 1940-1931.

4 Lamm DL, Riggs DR, Traynelis CL, Nseyo UO: Apparent failure of current intravesical chemotherapy prophylaxis to influence the long-term course of superficial transitional cell carcinoma of the bladder. J Urol 1995;153:1444-1450.

-5 Oddens J, Brausi M, Sylvester R, Bono A, van de Beek C, van Andel G, Gontero P, Hoeltl W, Turkeri L, Marreaud S, Collette S, Oosterlinck W: Final results of an EORTC-GU cancers group randomized study of maintenance bacillus Calmette-Guerin in intermediate- and high-risk Ta, T1 papillary carcinoma of the urinary bladder: one-third dose versus full dose and 1 year versus 3 years of maintenance. Eur Urol 2013;63:462-472.

6 Brunner YF, Rodriguez-Raecke R, Mutic S, Benedict C, Freiherr J: Neural correlates of olfactory and visual memory performance in 3D-simulated mazes after intranasal insulin application. Neurobiol Learn Mem 2016;134:256-263

7 van Valkenhoef G, Dias S, Ades AE, Welton NJ: Automated generation of node-splitting models for assessment of inconsistency in network meta-analysis. Res Synth Methods 2016;7:80-93.

-8 Addeo R, Caraglia M, Bellini S, Abbruzzese A, Vincenzi B, Montella L, Miragliuolo A, Guarrasi R, Lanna M, Cennamo G, Faiola V, Del Prete S: Randomized phase III trial on gemcitabine versus mytomicin in recurrent superficial bladder cancer: evaluation of efficacy and tolerance. J Clin Oncol 2010;28:543-548. 


\section{Cellular Physiology Cell Physiol Biochem 2017;41:1298-1312

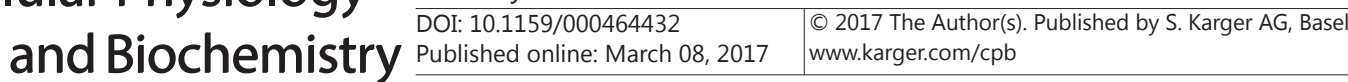

Wu et al.: Assessment of Therapies in Bladder Cancer

9 Ali-el-Dein B, Nabeeh A, el-Baz M, Shamaa S, Ashamallah A: Single-dose versus multiple instillations of epirubicin as prophylaxis for recurrence after transurethral resection of pTa and pT1 transitional-cell bladder tumours: a prospective, randomized controlled study. Br J Urol 1997;79:731-735.

10 Ali-El-Dein B, Nabeeh A, Ismail EH, Ghoneim MA: Sequential bacillus Calmette-Guerin and epirubicin versus bacillus Calmette-Guerin alone for superficial bladder tumors: A randomized prospective study. J Urol 1999;162:339-342.

-11 Bendary L, Khalil S, Shahin A, Nawar N: 1655 INTRAVESICAL GEMCITABINE VERSUS BACILLUS CALMETTE-GUERIN ( BCG) IN TREATMENT OF NON-MUSCLE INVASIVE BLADDER CANCER: SHORT TERM COMPARATIVE STUDY. J Urol 2011;185:e664-e665.

12 Bilen CY, Özen H, Aki FT, Aygün C, Ekici S, Kendi S: Clinical experience with BCG alone versus BCG plus epirubicin. Int J Urol 2000; 7:206-209.

13 Bohle A, Leyh H, Frei C, Kuhn M, Tschada R, Pottek T, Wagner W, Knispel HH, von Pokrzywnitzki W, Zorlu F, Helsberg K, Lubben B, Soldatenkova V, Stoffregen C, Buttner H, Group SS: Single postoperative instillation of gemcitabine in patients with non-muscle-invasive transitional cell carcinoma of the bladder: a randomised, double-blind, placebo-controlled phase III multicentre study. Eur Urol 2009;56:495-503.

14 Cai T, Nesi G, Tinacci G, Zini E, Mondaini N, Boddi V, Mazzoli S, Bartoletti R: Can Early Single Dose Instillation of Epirubicin Improve Bacillus Calmette-Guerin Efficacy in Patients With Nonmuscle Invasive High Risk Bladder Cancer? Results From a Prospective, Randomized, Double-Blind Controlled Study. J Urol 2008;180:110-115.

15 Cheng CW, Chan SF, Chan LW, Chan CK, Ng CF, Cheung HY, Chan SY, Wong WS, Lai FM, To KF, Li ML: Twelveyear follow up of a randomized prospective trial comparing bacillus Calmette-Guerin and epirubicin as adjuvant therapy in superficial bladder cancer. Int J Urol 2005;12:449-455.

16 De Nunzio C, Carbone A, Albisinni S, Alpi G, Cantiani A, Liberti M, Tubaro A, Iori F: Long-term experience with early single mitomycin $C$ instillations in patients with low-risk non-muscle-invasive bladder cancer: prospective, single-centre randomised trial. World J Urol 2011;29:517-521.

17 de Reijke TM, Kurth KH, Sylvester RJ, Hall RR, Brausi M, van de Beek K, Landsoght KE, Carpentier P, European Organization for the R, Treatment of Cancer-Genito-Urinary G: Bacillus Calmette-Guerin versus epirubicin for primary, secondary or concurrent carcinoma in situ of the bladder: results of a European Organization for the Research and Treatment of Cancer--Genito-Urinary Group Phase III Trial (30906). J Urol 2005;173:405-409.

18 Di Lorenzo G, Perdona S, Damiano R, Faiella A, Cantiello F, Pignata S, Ascierto P, Simeone E, De Sio M, Autorino R: Gemcitabine versus bacille Calmette-Guerin after initial bacille Calmette-Guerin failure in nonmuscle-invasive bladder cancer: a multicenter prospective randomized trial. Cancer 2010;116:1893-1900.

19 Di Stasi SM, Giannantoni A, Giurioli A, Valenti M, Zampa G, Storti L, Attisani F, De Carolis A, Capelli G, Vespasiani G, Stephen RL: Sequential BCG and electromotive mitomycin versus BCG alone for high-risk superficial bladder cancer: a randomised controlled trial. Lancet Oncol 2006;7:43-51.

-20 Di Stasi SM, Giannantoni A, Stephen RL, Capelli G, Navarra P, Massoud R, Vespasiani G: Intravesical electromotive mitomycin $\mathrm{C}$ versus passive transport mitomycin $\mathrm{C}$ for high risk superficial bladder cancer: a prospective randomized study. J Urol 2003;170:777-782.

21 El-Ghobashy S, El-Leithy TR, Roshdy MM, El-Ganzoury HM: Effectiveness of a single immediate mitomycin C instillation in patients with low risk superficial bladder cancer: short and long-term follow-up. J Egypt Natl Canc Inst 2007;19:121-126.

-22 Friedrich MG, Pichlmeier U, Schwaibold H, Conrad S, Huland H: Long-Term Intravesical Adjuvant Chemotherapy Further Reduces Recurrence Rate Compared with Short-Term Intravesical Chemotherapy and Short-Term Therapy with Bacillus Calmette-Guérin (BCG) in Patients with Non-Muscle-Invasive Bladder Carcinoma. Eur Urol 2007;52:1123-1130.

-23 Gontero P, Oderda M, Mehnert A, Gurioli A, Marson F, Lucca I, Rink M, Schmid M, Kluth LA, Pappagallo G, Sogni F, Sanguedolce F, Schiavina R, Martorana G, Shariat SF, Chun F: The impact of intravesical gemcitabine and $1 / 3$ dose Bacillus Calmette-Guerin instillation therapy on the quality of life in patients with nonmuscle invasive bladder cancer: results of a prospective, randomized, phase II trial. J Urol 2013;190:857-862.

24 Gudjonsson S, Adell L, Merdasa F, Olsson R, Larsson B, Davidsson T, Richthoff J, Hagberg G, Grabe M, Bendahl PO, Mansson W, Liedberg F: Should all patients with non-muscle-invasive bladder cancer receive early intravesical chemotherapy after transurethral resection? The results of a prospective randomised multicentre study. Eur Urol 2009;55:773-780. 


\section{Cellular Physiology Cell Physiol Biochem 2017;41:1298-1312 \begin{tabular}{l|l|l} 
and Biochemistry Publisned onine: IVIarch 08, 2017 & $\begin{array}{l}\text { @ 2017 The Author(s). Published by S. Karger AG, Basel } \\
\text { www.karger.com/cpb }\end{array}$ \\
\hline
\end{tabular}}

Wu et al.: Assessment of Therapies in Bladder Cancer

25 Gülpinar Ö, Halilioğlu AH, Gökçe MI, Göğüş Ç, Baltaci S: The value of perioperative mitomycin c instillation in improving subsequent bacillus calmette-guerin instillation efficacy in intermediate and high-risk patients with non-muscle invasive bladder cancer: A prospective randomized study. Int Braz J Urol 2012;38:474-479.

-26 Hinotsu S, Akaza H, Naito S, Ozono S, Sumiyoshi Y, Noguchi S, Yamaguchi A, Nagamori S, Terai A, Nasu Y, Kume H, Tomita Y, Tanaka Y, Samma S, Uemura H, Koga H, Tsushima T: Maintenance therapy with bacillus Calmette-Guérin Connaught strain clearly prolongs recurrence-free survival following transurethral resection of bladder tumour for non-muscle-invasive bladder cancer. BJU International 2011;108:187-195.

27 Inamoto T, Ubai T, Nishida T, Fujisue Y, Katsuoka Y, Azuma H: Comparable effect with minimal morbidity of low-dose Tokyo 172 strain compared with regular dose Connaught strain as an intravesical bacillus Calmette-Guerin prophylaxis in nonmuscle invasive bladder cancer: Results of a randomized prospective comparison. Urol Ann 2013;5:7-12.

-28 Irie A, Uchida T, Yamashita H, Matsumoto K, Satoh T, Koh H, Shimura S, Iwamura M, Baba S: Sufficient prophylactic efficacy with minor adverse effects by intravesical instillation of low-dose bacillus CalmetteGuérin for superficial bladder cancer recurrence. Int J Urol 2003;10:183-189.

29 Järvinen R, Kaasinen E, Rintala E: Long-term results of maintenance treatment of mitomycin C or alternating mitomycin $\mathrm{C}$ and bacillus Calmette-Guérin instillation therapy of patients with carcinoma in situ of the bladder: A subgroup analysis of the prospective FinnBladder 2 study with a 17-year follow-up. Scand J Urol Nephrol 2012;46:411-417.

-30 Jimenez-Cruz JF, Vera-Donoso CD, Leiva O, Pamplona M, Rioja-Sanz LA, Martinez-Lasierra M, Flores N, Unda M: Intravesical immunoprophylaxis in recurrent superficial bladder cancer (Stage T1): multicenter trial comparing bacille Calmette-Guerin and interferon-alpha. Urology 1997;50:529-535.

-31 Kolodziej A, Dembowski J, Zdrojowy R, Wozniak P, Lorenz J: Treatment of high-risk superficial bladder cancer with maintenance bacille Calmette-Guerin therapy: preliminary results. BJU Int 2002;89:620-622.

-32 Krege S, Giani G, Meyer R, Otto T, Rubben H: A randomized multicenter trial of adjuvant therapy in superficial bladder cancer: transurethral resection only versus transurethral resection plus mitomycin $\mathrm{C}$ versus transurethral resection plus bacillus Calmette-Guerin. Participating Clinics. J Urol 1996;156:962966.

-33 Kumar A, Dubey D, Bansal P, Mandhani A, Naik S: Urinary interleukin-8 predicts the response of standard and low dose intravesical bacillus calmette-guerin (modified Danish 1331 strain) for superficial bladder cancer. J Urol 2002;168:2232-2235.

-34 Lamm DL, Blumenstein BA, David Crawford E, Crissman JD, Lowe BA, Smith Jr JA, Sarosdy MF, Schellhammer PF, Sagalowsky AI, Messing EM, Loehrer P, Barton Grossman H: Randomized intergroup comparison of bacillus calmette-guerin immunotherapy and mitomycin $\mathrm{C}$ chemotherapy prophylaxis in superficial transitional cell carcinoma of the bladder a southwest oncology group study. Urol Oncol 1995;1:119-126.

-35 Librenjak D, Situm M, Eterovic D, Dogas Z, Gotovac J: Immunoprophylactic intravesical application of bacillus Calmette-Guerin after transurethral resection of superficial bladder cancer. Croat Med J 2003;44:187-192.

-36 Lundholm C, Norlen BJ, Ekman P, Jahnson S, Lagerkvist M, Lindeborg T, Olsson JL, Tveter K, Wijkstrom $\mathrm{H}$, Westberg R, Malmstrom PU: A randomized prospective study comparing long-term intravesical instillations of mitomycin C and bacillus Calmette-Guerin in patients with superficial bladder carcinoma. J Urol 1996;156:372-376.

-37 Malmström PU, Wijkström H, Lundholm C, Wester K, Busch C, Norlén BJ, Ekman P, Karlberg L, Ohlsson JO, Lindeborg T, Malmström PU, Norming U, Wijkström H, Susskind S, Sten J, Lagerkvist M, Jansson S, Tveter KJ: 5-Year followup of a randomized prospective study comparing mitomycin C and bacillus CalmetteGuerin in patients with superficial bladder carcinoma. J Urol 1999;161:1124-1127.

-38 March N, Narbon S, Urzaiz U, Calvo O, Molina R, Gomez F, Martin G, Gonzalez C, Piñeiro M: A multicenter and randomised prospective study comparing three intravesical therapies, two with bacillus calmetteguerin inmunotherapy and one with mitomicyn-C chemotherapy in medium and low risk superficial bladder tumours. Eur Urol Suppl 2003;2:190-190.

-39 Martinez-Pineiro JA, Flores N, Isorna S, Solsona E, Sebastian JL, Pertusa C, Rioja LA, Martinez-Pineiro L, Vela R, Camacho JE, Nogueira JL, Pereira I, Resel L, Muntanola P, Galvis F, Chesa N, De Torres JA, Carballido J, Bernuy C, Arribas S, Madero R, for C: Long-term follow-up of a randomized prospective trial comparing 


\section{Cellular Physiology Cell Physiol Biochem 2017;41:1298-1312 \begin{tabular}{l|l|l} 
and Biochemistry Publisned onine: IVIarch 08, 2017 & $\begin{array}{l}\text { @ 2017 The Author(s). Published by S. Karger AG, Basel } \\
\text { www.karger.com/cpb }\end{array}$ \\
\hline
\end{tabular}}

Wu et al.: Assessment of Therapies in Bladder Cancer

a standard $81 \mathrm{mg}$ dose of intravesical bacille Calmette-Guerin with a reduced dose of $27 \mathrm{mg}$ in superficial bladder cancer. BJU Int 2002;89:671-680.

-40 Melekos MD, Chionis HS, Paranychianakis GS, Dauaher HH: Intravesical 4'-epi-doxorubicin (epirubicin) versus bacillus Calmette-Guerin. A controlled prospective study on the prophylaxis of superficial bladder cancer. Cancer 1993;72:1749-1755.

41 Melekos MD, Zarakovitis I, Dandinis K, Fokaefs E, Chionis H, Dauaher H, Barbalias G: BCG versus epirubicin in the prophylaxis of multiple superficial bladder tumours: results of a prospective randomized study using modified treatment schemes. Int Urol Nephrol 1996;28:499-509.

-42 Melekos MD, Zarakovitis IE, Fokaefs ED, Dandinis K, Chionis H, Bouropoulos C, Dauaher H: Intravesical bacillus Calmette-Guerin versus epirubicin in the prophylaxis of recurrent and/or multiple superficial bladder tumours. Oncology 1996;53:281-288.

-43 Mondal HP, Yirang K, Mukhopadhyay C, Adhikary SS, Dutta B, Bhoj SS: Prospective randomized study between intravesical BCG and mitomycin-C for non-muscleinvasive urothelial carcinoma of urinarybladder post TURBT. Bangladesh J Med Sci 2016;15:74-77.

44 O'Brien T, Ray E, Singh R, Coker B, Beard R: Prevention of bladder tumours after nephroureterectomy for primary upper urinary tract urothelial carcinoma: a prospective, multicentre, randomised clinical trial of a single postoperative intravesical dose of mitomycin C (the ODMIT-C Trial). Eur Urol 2011;60:703-710.

-45 Oddens J, Brausi M, Sylvester R, Bono A, Van De Beek C, Van Andel G, Gontero P, Hoeltl W, Turkeri L, Marreaud S, Collette S, Oosterlinck W: Final results of an EORTC-GU cancers group randomized study of maintenance bacillus calmette-guérin in intermediate- and high-risk Ta, T1 papillary carcinoma of the urinary bladder: One-third dose versus full dose and 1 year versus 3 years of maintenance. Eur Urol 2013;63:462-472.

-46 Ojea A, Nogueira JL, Solsona E, Flores N, Gómez JMF, Molina JR, Chantada V, Camacho JE, Piñeiro LM, Rodríguez RH, Isorna S, Blas M, Martínez-Piñeiro JA, Madero R: A Multicentre, Randomised Prospective Trial Comparing Three Intravesical Adjuvant Therapies for Intermediate-Risk Superficial Bladder Cancer: Low-Dose Bacillus Calmette-Guerin (27 mg) versus Very Low-Dose Bacillus Calmette-Guerin (13.5 mg) versus Mitomycin C. Eur Urol 2007;52:1398-1406.

-47 Oosterlinck W, Kirkali Z, Sylvester R, Silva FCD, Busch C, Algaba F, Collette S, Bono A: Sequential intravesical chemoimmunotherapy with mitomycin $\mathrm{C}$ and bacillus Calmette-Guérin and with Bacillus Calmette-Guérin alone in patients with carcinoma in situ of the urinary bladder: Results of an EORTC genito-urinary group randomized phase 2 trial (30993). Eur Urol 2011;59:438-446.

48 Oosterlinck W, Kurth KH, Schroder F, Bultinck J, Hammond B, Sylvester R: A prospective European Organization for Research and Treatment of Cancer Genitourinary Group randomized trial comparing transurethral resection followed by a single intravesical instillation of epirubicin or water in single stage Ta, T1 papillary carcinoma of the bladder. J Urol 1993;149:749-752.

49 Peyromaure M, Zerbib M: T1G3 transitional cell carcinoma of the bladder: recurrence, progression and survival. BJU Int 2004;93:60-63.

50 Porena M, Del Zingaro M, Lazzeri M, Mearini L, Giannantoni A, Bini V, Costantini E: Bacillus CalmetteGuerin versus gemcitabine for intravesical therapy in high-risk superficial bladder cancer: a randomised prospective study. Urol Int 2010;84:23-27.

51 Rajala P, Kaasinen E, Raitanen M, Liukkonen T, Rintala E: Perioperative single dose instillation of epirubicin or interferon-alpha after transurethral resection for the prophylaxis of primary superficial bladder cancer recurrence: a prospective randomized multicenter study--FinnBladder III long-term results. J Urol 2002;168:981-985.

52 Rajala P, Liukkonen T, Raitanen M, Rintala E, Kaasinen E, Helle M, Lukkarinen O: Transurethral resection with perioperative instilation on interferon-alpha or epirubicin for the prophylaxis of recurrent primary superficial bladder cancer: a prospective randomized multicenter study--Finnbladder III. J Urol 1999;161:1133-1135;discussion 1135-1136.

53 Rintala E, Jauhiainen K, Kaasinen E, Nurmi M, Alfthan O, Hansson E, Juusela H, Kanerva K, Korhonen H, Permi J, Petäys P, Puolakka VM, Rajala P, Ruutu M, Tainio H, Talja M, Tuhkanen K, Viitanen J: Alternating mitomycin C and bacillus Calmette-Guerin instillation prophylaxis for recurrent papillary (stages Ta to T1) superficial bladder cancer. J Urol 1996;156:56-60. 


\section{Cellular Physiology Cell Physiol Biochem 2017;41:1298-1312 \begin{tabular}{l|l} 
and Biochemistry Publisned onIme: IVIarcn 08, 2017 & $\begin{array}{l}\text { ( ) 2017 The Author(s). Published by S. Karger AG, Basel } \\
\text { www.karger.com/cpb }\end{array}$
\end{tabular}}

Wu et al.: Assessment of Therapies in Bladder Cancer

54 Solsona E, Iborra I, Ricos JV, Monros JL, Casanova J, Dumont R: Effectiveness of a single immediate mitomycin C instillation in patients with low risk superficial bladder cancer: short and long-term followup. J Urol 1999;161:1120-1123.

-55 Solsona E, Madero R, Chantada V, Fernandez JM, Zabala JA, Portillo JA, Alonso JM, Astobieta A, Unda M, Martinez-Pineiro L, Rabadan M, Ojea A, Rodriguez-Molina J, Beardo P, Muntanola P, Gomez M, Montesinos $M$, Martinez Pineiro JA: Sequential combination of mitomycin C plus bacillus Calmette-Guerin (BCG) is more effective but more toxic than BCG alone in patients with non-muscle-invasive bladder cancer in intermediate- and high-risk patients: final outcome of CUETO 93009, a randomized prospective trial. Eur Urol 2015;67:508-516.

-56 Sylvester RJ, Brausi MA, Kirkels WJ, Hoeltl W, Calais Da Silva F, Powell PH, Prescott S, Kirkali Z, van de Beek C, Gorlia T, de Reijke TM, Group EG-UTC: Long-term efficacy results of EORTC genito-urinary group randomized phase 3 study 30911 comparing intravesical instillations of epirubicin, bacillus CalmetteGuerin, and bacillus Calmette-Guerin plus isoniazid in patients with intermediate- and high-risk stage Ta T1 urothelial carcinoma of the bladder. Eur Urol 2010;57:766-773.

57 Takashi M, Kakai K, Ohno Y, Murase T, Miyake K: Evaluation of a low-dose intravesical bacillus CalmetteGuérin (Tokyo strain) therapy for superficial bladder cancer. Int Urol Nephrol 1995;27:723-733.

-58 Tolley DA, Hargreave TB, Smith PH, Williams JL, Grigor KM, Parmar MK, Freedman LS, Uscinska BM: Effect of intravesical mitomycin $\mathrm{C}$ on recurrence of newly diagnosed superficial bladder cancer: interim report from the Medical Research Council Subgroup on Superficial Bladder Cancer (Urological Cancer Working Party). Br Med J (Clin Res Ed) 1988;296:1759-1761.

59 Tozawa K, Okamura T, Sasaki S, Kawai N, Ito Y, Hayashi Y, Kohri K: Intravesical combined chemoimmunotherapy with epirubicin and bacillus Calmette-Guérin is not indicated for superficial bladder cancer. Urol Int 2001;67:289-292.

60 van der Meijden AP, Brausi M, Zambon V, Kirkels W, de Balincourt C, Sylvester R, Members of the EG-UG: Intravesical instillation of epirubicin, bacillus Calmette-Guerin and bacillus Calmette-Guerin plus isoniazid for intermediate and high risk Ta, T1 papillary carcinoma of the bladder: a European Organization for Research and Treatment of Cancer genito-urinary group randomized phase III trial. J Urol 2001;166:476481.

61 Vegt PD, Witjes JA, Witjes WP, Doesburg WH, Debruyne FM, van der Meijden AP: A randomized study of intravesical mitomycin C, bacillus Calmette-Guerin Tice and bacillus Calmette-Guerin RIVM treatment in pTa-pT1 papillary carcinoma and carcinoma in situ of the bladder. J Urol 1995;153:929-933.

62 Witjes JA, Caris CTM, Mungan NA, Debruyne FMJ, Witjes WPJ: Results of a randomized phase III trial of sequential intravesical therapy with mitomycin $\mathrm{C}$ and bacillus Calmette-Guerin versus mitomycin $\mathrm{C}$ alone in patients with superficial bladder cancer. J Urol 1999;160:1668-1672.

63 Wu WJ, Ke HL, Yang YH, Li CC, Chou YH, Huang CH: Should patients with primary upper urinary tract cancer receive prophylactic intravesical chemotherapy after nephroureterectomy? J Urol 2010;183:56-61.

64 Yoneyama T, Ohyama C, Imai A, Ishimura H, Hagisawa S, Iwabuchi I, Mori K, Kamimura N, Koie T, Yamato T, Suzuki T: Low-Dose Instillation Therapy with Bacille Calmette-Guérin Tokyo 172 Strain After Transurethral Resection: Historical Cohort Study. Urology 2008;71:1161-1165.

65 Crispen R: History of BCG and its substrains. Prog Clin Biol Res 1989;310:35-50.

66 van der Meijden AP, Debruyne FM, Steerenberg PA, de Jong WH: Aspects of non-specific immunotherapy with BCG in superficial bladder cancer: an overview. Prog Clin Biol Res 1989;310:11-33.

67 Lamm DL, Thor DE, Harris SC, Reyna JA, Stogdill VD, Radwin HM: Bacillus Calmette-Guerin immunotherapy of superficial bladder cancer. J Urol 1980;124:38-40.

-68 Herr HW, Wartinger DD, Fair WR, Oettgen HF: Bacillus Calmette-Guerin therapy for superficial bladder cancer: a 10-year followup. J Urol 1992;147:1020-1023.

69 Martinez-Pineiro JA, Martinez-Pineiro L, Solsona E, Rodriguez RH, Gomez JM, Martin MG, Molina JR, Collado AG, Flores N, Isorna S, Pertusa C, Rabadan M, Astobieta A, Camacho JE, Arribas S, Madero R, Club Urologico Espanol de Tratamiento 0: Has a 3-fold decreased dose of bacillus Calmette-Guerin the same efficacy against recurrences and progression of T1G3 and Tis bladder tumors than the standard dose? Results of a prospective randomized trial. J Urol 2005;174:1242-1247.

70 Bilen CY, Ozen H, Aki FT, Aygun C, Ekici S, Kendi S: Clinical experience with BCG alone versus BCG plus epirubicin. Int J Urol 2000;7:206-209. 